JOURNAL OF THE

AMERICAN MATHEMATICAL SOCIETY

Volume 17, Number 2, Pages 443-471

S 0894-0347(04)00452-7

Article electronically published on February 3, 2004

\title{
GEOMETRIC CONTROL IN THE PRESENCE OF A BLACK BOX
}

\author{
NICOLAS BURQ AND MACIEJ ZWORSKI
}

\section{INTRODUCTION}

The purpose of this paper is to show how ideas coming from scattering theory (resolvent estimates) lead to results in control theory and to some closely related eigenfunction estimates.

The black box approach in scattering theory developed by Sjöstrand and the second author [37] puts scattering problems with different structures in one framework and allows abstract applications of spectral results known for confined systems. One striking example is a reduction of scattering on finite volume surfaces to one dimensional black box scattering. In this paper we take the opposite point of view: a black box in a confined system is replaced by a scattering problem. That permits having isolated dynamical phenomena (such as only one closed orbit) impossible in confined systems. It also permits using some finer results of scattering theory directly.

We stress that this follows the well-established trend (see Bardos-Lebeau-Rauch 2]) of using propagation of singularities results developed for scattering theory in geometric control theory. We also mention that the term "black box" is commonly used, in a similar context, in applied control theory [39].

Since the proofs are simple and since it is profitable to state the results in an abstract setting which requires a certain amount of preparation, in this section we will present some typical applications.

In geometric control theory for the Schrödinger equation (see Lebeau [29], and also [30], [44] for earlier work and background) we are concerned with the following mixed problem:

$$
\begin{aligned}
& \left(i \partial_{t}+\Delta\right) u=0 \quad \text { in } \Omega, \\
& \left.u\right|_{[0, T] \times \Omega}=g \mathbb{1}_{[0, T] \times \Gamma}, \\
& \left.u\right|_{t=0}=u_{0},
\end{aligned}
$$

where $\Omega$ is an open subset of $\mathbb{R}^{d}, \partial \Omega$ is its boundary and $\Gamma$ is an open subset of $\partial \Omega$. The question is to determine a (large) class of functions $u_{0}$ for which there exists a control $g$ such that $\left.u\right|_{t=T}=0$. In a geometric setting in which full geometric control fails, the following result was established by the first author in [5]:

Theorem 1. Consider $\Theta=\bigcup_{j=1}^{N} \Theta_{j} \subset \mathbb{R}^{d}$, a union of mutually disjoint closed sets with strictly convex smooth boundaries and satisfying the assumptions in subsection

Received by the editors May 14, 2003.

2000 Mathematics Subject Classification. Primary 35B37, 35P20, 81Q20.

(C)2004 American Mathematical Society 
6.2 below. Let $\widetilde{\Omega}$ be a bounded domain with a smooth boundary and containing convhull $(\Theta)$. Denote by $\Omega=\widetilde{\Omega} \backslash \Theta$ and $\Gamma=\partial \widetilde{\Omega}$. Then for any $T, \varepsilon>0$ and any $u_{0} \in H_{0}^{1+\varepsilon}(\Omega)$ there exists $g \in L^{2}([0, T] \times \Gamma)$ such that in (1.1) we have $\left.u\right|_{t>T} \equiv 0$.

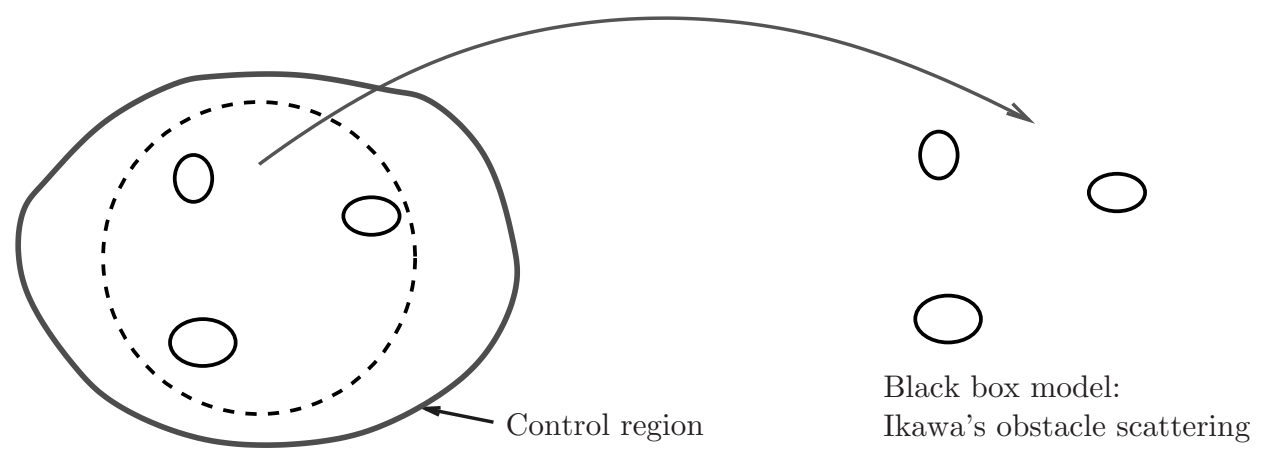

Figure 1. Control in the exterior of several convex bodies.

In Figure 1 on the left we have three convex obstacles inside of the boundary of $\widetilde{\Omega}$. Inside of the black box bounded by the dotted line the local geometry is the same as in the scattering problem on the right.

We are going to show how Theorem 1 can be obtained directly from estimates on the resolvent of the Laplace operator, which in turn can be deduced from semiclassical microlocal analysis or from known results in scattering theory. In the case quoted above, these come from the work of Ikawa [26] and in particular we can now avoid most of the delicate analysis of [5].

The next application generalizes a result of Colin de Verdière and Parisse [13] who considered a special case of an isolated trajectory lying on a segment of a constant negative curvature cylinder in dimension two:

Theorem 2. Suppose that $(X, g)$ is a compact Riemannian manifold with a (possibly empty) boundary and $\gamma \subset X$ is a closed real hyperbolic geodesic not intersecting the boundary. If $\chi \in \mathcal{C}^{\infty}(X,[0,1])$ is supported in a sufficiently small neighbourhood of $\gamma$, then there exists a constant $C=C(\gamma)$ such that for any eigenfunction, $u$, of the Laplacian, $\Delta_{g}$ with Dirichlet or Neumann boundary conditions, we have

$$
C \int_{X}|u(x)|^{2}(1-\chi)(x) d \operatorname{vol}_{g} \geq \frac{1}{\log \lambda} \int_{X}|u(x)|^{2} d \operatorname{vol}_{g}, \quad-\Delta_{g} u=\lambda u .
$$

An example 13 of a cylinder segment with Dirichlet boundary conditions shows that the result is optimal. The theorem remains true if we allow broken geodesic flow as long as the reflections are all transversal. To make the exposition selfcontained (in particular, the derivation of the needed estimates in the Appendix), we present the result in the simpler case.

The proof of Theorem 2 (see also Theorem 2]) is based on putting the closed hyperbolic orbit into a microlocal black box, where that orbit becomes the only trapped orbit in a scattering problem. We can then use scattering estimates based on the work of Gérard [17] and Gérard-Sjöstrand [18] to obtain estimates leading to (1.2). When the geodesic does not hit the boundary, we present a self-contained argument where in place of a scattering black box we use a complex absorbing potential. 


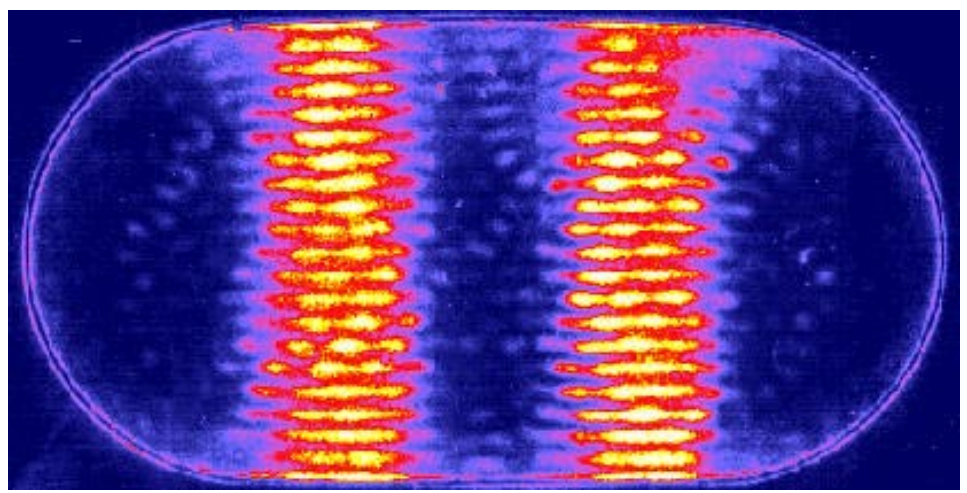

Figure 2. An experimental image of the wave in the "black box" in Figure [5; see [11] and http://www.bath.ac.uk/ pyscmd/ acoustics.

We conclude with a brief discussion of another example related to eigenvalue scarring (see Theorem 9 below for a full discussion). While in Theorem 2 we eliminated the need for separation of variables, its use is essential in this case. For the Bunimovich cavity shown in Figure 2 the natural black box for constructing bouncing ball modes (two are shown in the same figure) is a rectangle constituting the central part of the cavity - see the recent discussion of this in [16] and [42. On one hand, our result shows that the crude error estimate

$$
\left(-\Delta_{D}-\lambda\right) u_{\lambda}=\mathcal{O}(1), \quad\left\|u_{\lambda}\right\|=1,
$$

for the quasimodes obtained by truncating the rectangle modes is in fact the best possible and on the other hand that the eigenfunctions cannot accumulate at high frequency only in the central part. This agrees with the experimental results [11] where it was stressed that phenomena shown in Figure 2 can occur only at low frequencies (see also [1] for a different discussion and references to the physics literature). For an exact eigenstate we have the following

Theorem 3. Let u be a Dirichlet eigenfunction of the Laplacian on the Bunimovich stadium $M$ :

$$
-\Delta u=\lambda u,\left.\quad u\right|_{\partial M}=0
$$

Let $a(x)$ be any continuous function identically 1 on the nonrectangular part of $M$. Then there exists $C>0$ such that

$$
C \int_{M}|a(x) u(x)|^{2} d x \geq \int_{M}|u(x)|^{2} d x .
$$

Stronger results (implying (1.4) are presented in Theorems 3 and 9 in subsection 6.3. A self-contained proof of Theorem 3 and a discussion of related mathematical and physical literature has been presented in [10. We stress that only the properties of the rectangular part used as a "black box" are needed for this result.

\section{Preliminaries}

In this section we review some basic aspects of semi-classical microlocal analysis, following [38, Section 3]. Thus, let $X$ be a compact $\mathcal{C}^{\infty}$ manifold. We consider pseudodifferential operators as acting on half-densities, $u(x)|d x|^{\frac{1}{2}} \in \mathcal{C}^{\infty}\left(X, \Omega_{X}^{\frac{1}{2}}\right)$, 
where we use the informal notation indicating how the half-densities change under changes of variables:

$$
u(x)|d x|^{\frac{1}{2}}=v(y)|d y|^{\frac{1}{2}}, \quad y=\kappa(x) \Longleftrightarrow v(\kappa(x))\left|\kappa^{\prime}(x)\right|^{\frac{1}{2}}=u(x),
$$

Consequently the symbols will also be considered as half-densities; see [24 Section 18.1] for a general introduction and [38, Appendix] for a discussion of the semiclassical case. This way our results are more general and do not depend on the choice of a metric on $X$. If $X$ is a Riemannian manifold and the operator we consider is its Laplace-Bertrami operator, then the natural Riemannian density is all we need.

By symbols on $X$ we mean the following class:

$$
\begin{aligned}
& S^{k, m}\left(T^{*} X, \Omega_{T^{*} X}^{\frac{1}{2}}\right)=\left\{a \in \mathcal{C}^{\infty}\left(T^{*} X \times(0,1], \Omega_{T^{*} X}^{\frac{1}{2}}\right):\right. \\
&\left.\left|\partial_{x}^{\alpha} \partial_{\xi}^{\beta} a(x, \xi, h)\right| \leq C_{\alpha, \beta} h^{-m}\langle\xi\rangle^{k-|\beta|}\right\},
\end{aligned}
$$

and the class of corresponding pseudodifferential operators, $\Psi_{h}^{m, k}\left(X, \Omega_{X}^{\frac{1}{2}}\right)$, obtained from a local formula in $\mathbb{R}^{n}$ :

$$
\mathrm{Op}_{h}^{w}(a) u(x)=\frac{1}{(2 \pi h)^{n}} \iint a\left(\frac{x+y}{2}, \xi, h\right) e^{i\langle x-y, \xi\rangle / h} u(y) d y d \xi .
$$

The principal symbol map,

$$
\sigma_{h}: \Psi_{h}^{m, k}\left(X, \Omega_{X}^{\frac{1}{2}}\right) \longrightarrow S^{k, m} / S^{k-1, m-1}\left(T^{*} X, \Omega_{T^{*} X}^{\frac{1}{2}}\right),
$$

gives the left inverse of $\mathrm{Op}_{h}^{w}$ in the sense that $\sigma_{h} \circ \mathrm{Op}_{h}^{w}: S^{m, k} \rightarrow S^{m, k} / S^{m-1, k-1}$ is the natural projection. We refer to [15] for a detailed discussion of the Weyl quantization and to [41] for a discussion in the case of manifolds.

When acting on half-densities, the principal symbol is in fact well defined in $S^{k, m} / S^{k-2, m-2}$, that is, up to $\mathcal{O}\left(h^{2}\right)$ as far as the order in $h$ is concerned; see 38, Appendix]. When $P(h)$ is polyhomogeneous, that is, $P(h)=O p(P(\bullet, h))$, and $P(x, \xi, h) \sim p_{0}(x, \xi)+h p_{1}(x, \xi)+\cdots, p_{j} \in S^{k-j, m}$, the subprincipal symbol,

$$
p_{1}^{s}(x, \xi)=p_{1}(x, \xi),
$$

is well defined. In general, we will say that the subprincipal symbol of $P(h) \in$ $\Psi_{h}^{m, k}\left(X, \Omega_{X}^{\frac{1}{2}}\right)$ vanishes if the principal symbol of $P(h)$ in $S^{k, m} / S^{k-2, m-2}$ is independent of $h$.

For $a \in S^{m, k}\left(T^{*} X, \Omega_{T^{*} X}^{\frac{1}{2}}\right)$ we follow 38 , in defining

$$
\operatorname{ess-Supp}_{h} a \subset T^{*} X \sqcup S^{*} X, \quad S^{*} X \stackrel{\text { def }}{=}\left(T^{*} X \backslash 0\right) / \mathbb{R}_{+},
$$

where the usual $\mathbb{R}_{+}$action is given by multiplication on the fibers: $(x, \xi) \mapsto(x, t \xi)$, as

$$
\begin{aligned}
& \operatorname{ess}^{\operatorname{supp}_{h}} a= \\
& \complement\left\{(x, \xi) \in T^{*} X: \exists \epsilon>0 \partial_{x}^{\alpha} \partial_{\xi}^{\beta} a\left(x^{\prime}, \xi^{\prime}\right)=\mathcal{O}\left(h^{\infty}\right), d\left(x, x^{\prime}\right)+\left|\xi-\xi^{\prime}\right|<\epsilon\right\} \\
& \cup \complement\left\{(x, \xi) \in T^{*} X \backslash 0: \exists \epsilon>0 \partial_{x}^{\alpha} \partial_{\xi}^{\beta} a\left(x^{\prime}, \xi^{\prime}\right)=\mathcal{O}\left(h^{\infty}\left\langle\xi^{\prime}\right\rangle^{-\infty}\right),\right. \\
& \left.\quad d\left(x, x^{\prime}\right)+1 /\left|\xi^{\prime}\right|+|\xi /| \xi\left|-\xi^{\prime} /\right| \xi^{\prime}||<\epsilon\right\} / \mathbb{R}_{+} .
\end{aligned}
$$

For $A \in \Psi_{h}^{m, k}\left(X, \Omega_{X}^{\frac{1}{2}}\right)$, put

$$
W F_{h}(A)=\operatorname{ess} \operatorname{supp}_{h} a, \quad A=\mathrm{Op}_{h}^{w}(a),
$$


and this definition does not depend on the choice of $\mathrm{Op}_{h}^{w}$. For

$$
u \in \mathcal{C}^{\infty}\left((0,1]_{h}, \mathcal{D}^{\prime}\left(X, \Omega_{X}^{\frac{1}{2}}\right)\right), \quad \exists N_{0}, \quad h^{N_{0}} u \text { is bounded in } \mathcal{D}^{\prime}\left(X, \Omega_{X}^{\frac{1}{2}}\right),
$$

we define the semi-classical wave front set as

$$
\begin{array}{r}
W F_{h}(u)=\complement\left\{(x, \xi): \exists A \in \Psi_{h}^{0,0}\left(X, \Omega_{X}^{\frac{1}{2}}\right) \sigma_{h}(A)(x, \xi) \neq 0,\right. \\
\left.A u \in h^{\infty} \mathcal{C}^{\infty}\left((0,1]_{h}, \mathcal{C}^{\infty}\left(X, \Omega_{X}^{\frac{1}{2}}\right)\right)\right\} .
\end{array}
$$

When $u$ is not necessarily smooth, we can give a definition analogous to that of $\operatorname{ess-supp}_{h} a$. In this paper we will work in a pure semi-classical setting and consequently only compact subsets of $T^{*} X$ will be important. Consequently, this definition is sufficient for our purposes.

We also need to review the notion of microlocal equivalence of operators and other objects. Suppose that

$$
T: \mathcal{C}^{\infty}\left(X, \Omega_{X}^{\frac{1}{2}}\right) \rightarrow \mathcal{C}^{\infty}\left(X, \Omega_{X}^{\frac{1}{2}}\right)
$$

and that for any semi-norm $\|\bullet\|_{1}$ on $\mathcal{C}^{\infty}\left(X, \Omega_{X}^{\frac{1}{2}}\right)$ there exists a semi-norm $\|\bullet\|_{2}$ and $M_{0}$ such that

$$
\|T u\|_{1}=\mathcal{O}\left(h^{-M_{0}}\right)\|u\|_{2}
$$

This condition makes $T$ semi-classically tempered. In the sequel all operators considered will be assumed to satisfy this temperance condition. For open precompact sets, $V \subset T^{*} X, U \subset T^{*} X$, the operators defined microlocally near $V \times U$ are given by equivalence classes of tempered operators given by the relation

$$
T \sim T^{\prime} \Longleftrightarrow A\left(T-T^{\prime}\right) B=\mathcal{O}\left(h^{\infty}\right): \mathcal{D}^{\prime}\left(X, \Omega_{X}^{\frac{1}{2}}\right) \longrightarrow \mathcal{C}^{\infty}\left(X, \Omega_{X}^{\frac{1}{2}}\right),
$$

for any $A, B \in \Psi_{h}^{0,0}\left(X, \Omega_{X}^{\frac{1}{2}}\right)$ such that

$$
\begin{aligned}
& W F(A) \subset \widetilde{V}, \quad W F(B) \subset \widetilde{U}, \\
& \quad \bar{V} \Subset \widetilde{V} \Subset T^{*} X, \quad \bar{U} \Subset \widetilde{U} \Subset T^{*} X, \widetilde{U}, \widetilde{V} \quad \text { open } .
\end{aligned}
$$

We say that $P=Q$ microlocally near $U \times V$ if $A P B-A Q B=\mathcal{O}_{L^{2} \rightarrow L^{2}}\left(h^{\infty}\right)$, where because of the assumed precompactness of $U$ and $V$ the $L^{2}$ norms can be replaced by any other norms. For operator identities this will be the meaning of equality of operators in this paper, with $U, V$ specified (or clear from the context). Similarly, we say that $B=T^{-1}$ microlocally near $V \times V$ if $B T=I$ microlocally near $U \times U$ and $T B=I$ microlocally near $V \times U$. More generally, we could say that $P=Q$ microlocally on $W \subset T^{*} X \times T^{*} X$ (or, say, $P$ is microlocally defined there) if for any $U, V, U \times V \subset W, P=Q$ microlocally in $U \times V$. We should stress that "microlocally" is always meant in this semi-classical sense in our paper.

Rather than review the definition of $h$-Fourier integral operators, we will recall a characterization which is essentially a converse of Egorov's theorem:

Proposition 2.1. Suppose that $U=\mathcal{O}(1): L^{2}(X) \rightarrow L^{2}(X)$ and that for every $A \in \Psi_{h}^{0,0}(X)$ we have

$$
A U=U B, \quad B \in \Psi_{h}^{0,0}(X), \quad \sigma(B)=\kappa^{*} \sigma(A),
$$

microlocally near $\left(m_{0}, m_{0}\right)$ where $\kappa: T^{*} X \rightarrow T^{*} X$ is a symplectomorphism, defined locally near $m_{0}, \kappa\left(m_{0}\right)=m_{0}$. Then $U$ is, microlocally, near $\left(m_{0}, m_{0}\right)$, an $h$-Fourier integral operator of order zero, quantizing $\kappa$, that is associated to the graph of $\kappa$. 
For the proof and further details we refer the reader to [38, Lemma 3.4]. We will use the following well-known fact (see [38, Proposition 3.5] for the proof):

Proposition 2.2. Suppose that $P \in \Psi_{h}^{0, k}(X)$ has a real principal symbol which satisfies the condition

$$
p=0 \Longrightarrow d p \neq 0 \text {. }
$$

For any $m_{0} \in p^{-1}(0)$ there exists an h-Fourier integral operator, $F$,

$$
\begin{gathered}
F P=h D_{x_{1}} F, \text { microlocally near }\left((0,0), m_{0}\right), \\
F^{-1} \text { exists microlocally near }\left(m_{0},(0,0)\right) .
\end{gathered}
$$

\section{From Resolvent estimates to time dependent control}

In this section we will present a simple abstract argument showing how semiclassical resolvent estimates give a control result for the semi-classical Schrödinger operator. An adaptation of this argument to the classical control setting will be presented in Section 5 That section will also provide a motivation for this type of estimates.

Theorem 4. Let $P(h)$ be a family of self-adjoint operators on a Hilbert space $\mathcal{H}$, with a fixed domain $\mathcal{D}$. Let $\mathcal{H}_{1}$ be another Hilbert space, and suppose that for a bounded family of operators, $A(h): \mathcal{D} \rightarrow \mathcal{H}_{1}$, we have

$$
\|u\|_{\mathcal{H}} \leq \frac{G(h)}{h}\|(P(h)+\tau) u\|_{\mathcal{H}}+g(h)\|A(h) u\|_{\mathcal{H}_{1}},
$$

for all $\tau \in I=(-b,-a) \Subset \mathbb{R}, 1 \leq G(h)=\mathcal{O}\left(h^{-N_{0}}\right)$, for some $N_{0}$. Fix $\chi \in$ $\mathcal{C}_{\mathrm{c}}^{\infty}((a, b))$. There exist constants $c_{0}, C_{0}$ and $h_{0}>0$ such that for any $T(h)$ satisfying

$$
\frac{G(h)}{T(h)}<c_{0}
$$

we have for $0<h<h_{0}$,

$$
\|\chi(P(h)) u\|_{\mathcal{H}}^{2} \leq C_{0} \frac{g(h)^{2}}{T(h)} \int_{0}^{T(h)}\left\|A(h) e^{-i t P(h) / h} \chi(P(h)) u\right\|_{\mathcal{H}_{1}}^{2} d t .
$$

To motivate the abstract presentation, we relate the notation of Theorem 4 to a concrete situation. Thus let $P(h)=-h^{2} \Delta$ be the Dirichlet Laplacian on a compact manifold $\Omega$, with boundary $\partial \Omega$. Then

$$
\mathcal{H}=L^{2}(\Omega), \quad \mathcal{D}=H^{2}(\Omega) \cap H_{0}^{1}(\Omega) .
$$

Let $\Gamma \subset \partial \Omega$. We then define

$$
\mathcal{H}_{1}=L^{2}(\Gamma), \quad \mathcal{D} \ni u \longmapsto A(h) u=\left.h \partial_{\nu} u\right|_{\Gamma} \in \mathcal{H}_{1},
$$

where $\partial_{\nu}$ denotes the inward pointing normal to $\partial \Omega$. The estimate (3.3) is a typical observability estimate equivalent by duality to an exact control statement (see subsection 6.1). An abstract method for obtaining semi-classical estimates (3.1) will be presented in Section 4 .

Proof. Let us put $v(t)=\exp (-i t P(h) / h) \chi(P(h)) u$. We introduce a function $\psi \in$ $\mathcal{C}_{\mathrm{c}}^{\infty}(\mathbb{R},[0,1])$ and put

$$
w(t)=\psi\left(\frac{t}{T(h)}\right) v(t) .
$$


Clearly,

$$
\left(i h \partial_{t}-P\right) w(t)=\frac{i h}{T(h)} \psi^{\prime}\left(\frac{t}{T(h)}\right) v(t) .
$$

Because of the compact support, we can take the (semi-classical) Fourier transform in $t$ which gives

$$
(\tau+P) \widehat{w}(\tau)=-\frac{i h}{T(h)} \mathcal{F}_{t \rightarrow \tau}\left(\psi^{\prime}(\bullet / T(h)) v\right)(\tau) .
$$

For $\tau \in I$ we can use (3.1) which gives

$$
\|\widehat{w}(\tau)\|_{\mathcal{H}} \leq \frac{G(h)}{T(h)}\left\|\mathcal{F}_{t \rightarrow \tau}\left(\psi^{\prime}(\bullet / T(h)) v\right)(\tau)\right\|_{\mathcal{H}}+g(h)\|A(h) \widehat{w}(\tau)\|_{\mathcal{H}_{1}} .
$$

Using the generalized Plancherel theorem, we obtain

$$
\int_{I}\|\widehat{w}(\tau)\|_{\mathcal{H}}^{2} d \tau \leq 2 \frac{G(h)^{2}}{T(h)^{2}}\left\|\psi^{\prime}(\bullet / T(h)) v\right\|_{L^{2}\left(\mathbb{R}_{t}, \mathcal{H}\right)}^{2}+2 g(h)^{2}\|A(h) w\|_{L^{2}\left(\mathbb{R}_{t}, \mathcal{H}_{1}\right)}^{2} .
$$

We now want to show that we can integrate over $\mathbb{R}$ in place of $I$ in the left-hand side. That follows from

$$
\left\|\hat{w}(\tau) \mathbb{1}_{\mathbb{R} \backslash I}(\tau)\right\|_{\mathcal{H}}=\mathcal{O}\left(\left(\frac{h}{1+|\tau|}\right)^{\infty}\right)\|\chi(P) u\|_{\mathcal{H}},
$$

which in turn follows from integration by parts in

$$
\begin{aligned}
\hat{w}(\tau) & =\int_{\mathbb{R}} e^{-i t(P+\tau) / h} \psi\left(\frac{t}{T}\right) \chi(P) u d t \\
& =\int_{\mathbb{R}}\left(-(P+\tau)^{-1} h D_{t} e^{-i t(P+\tau) / h}\right) \psi\left(\frac{t}{T}\right) \chi(P) u d t,
\end{aligned}
$$

using

$$
\forall \tau \in \mathbb{R} \backslash I \quad\|(P+\tau) \chi(P) u\|_{\mathcal{H}} \geq \frac{1}{C}\|\chi(P) u\|_{\mathcal{H}}
$$

Thus we obtained

$$
\begin{array}{rl}
\|w\|_{L^{2}\left(\mathbb{R}_{t}, \mathcal{H}\right) \leq}^{2} & 2 \frac{G(h)^{2}}{T(h)^{2}}\left\|\psi^{\prime}(\bullet / T(h)) v\right\|_{L^{2}\left(\mathbb{R}_{t}, \mathcal{H}\right)}^{2} \\
& +2 g(h)^{2}\|A(h) w\|_{L^{2}\left(\mathbb{R}_{t}, \mathcal{H}_{1}\right)}^{2}+\mathcal{O}\left(h^{\infty}\right)\|\chi(P) u\|^{2},
\end{array}
$$

and the first term on the right can be absorbed on the left using (3.2). In fact, since

$$
\sup _{\phi \in \mathcal{C}_{\mathrm{c}}^{\infty}((0,1))} \frac{\int_{0}^{1} \phi(s)^{2} d s}{\int_{0}^{1} \phi^{\prime}(s)^{2} d s}=\pi^{-2},
$$

we have from the definition of $w$, and for any $\epsilon>0$,

$$
\begin{aligned}
\|\chi(P) u\|_{\mathcal{H}}^{2} \leq & 2\left(\pi^{2}+\epsilon\right) \frac{G(h)^{2}}{T(h)^{2}}\|\chi(P) u\|_{\mathcal{H}}^{2} \\
& +2 \frac{g(h)^{2}}{T(h)}\|A(h) w\|_{L^{2}\left(\mathbb{R}_{t}, \mathcal{H}_{1}\right)}^{2}+\mathcal{O}\left(h^{\infty}\right)\|\chi(P) u\|_{\mathcal{H}}^{2} .
\end{aligned}
$$

This completes the proof once we take $h$ small enough.

It is a little surprising that any nonvanishing function $\psi$ with properties required in the proof will give an estimate with some constant, and consequently (3.5) is irrelevant. 


\section{Semiclassical BlaCK BOX RESOlvent EStimates}

In this section we will make assumptions under which resolvent estimates can be obtained in the semi-classical setting. For simplicity no boundary will be allowed here.

Let $X$ be a compact $\mathcal{C}^{\infty}$ manifold. Let $P(h) \in \Psi_{h}^{2,0}\left(X, \Omega_{X}^{\frac{1}{2}}\right)$ be formally selfadjoint on $L^{2}\left(X, \Omega_{X}^{\frac{1}{2}}\right)$. We assume that, if $p$ is the principal symbol of $P(h)$, then

$$
p=0 \Longrightarrow d p \neq 0, \quad p \geq\langle\xi\rangle^{2} / C \text { for }|\xi| \geq C,
$$

and that for some $\delta>0$

$$
p^{-1}([-\delta, \delta]) \Subset T^{*} X
$$

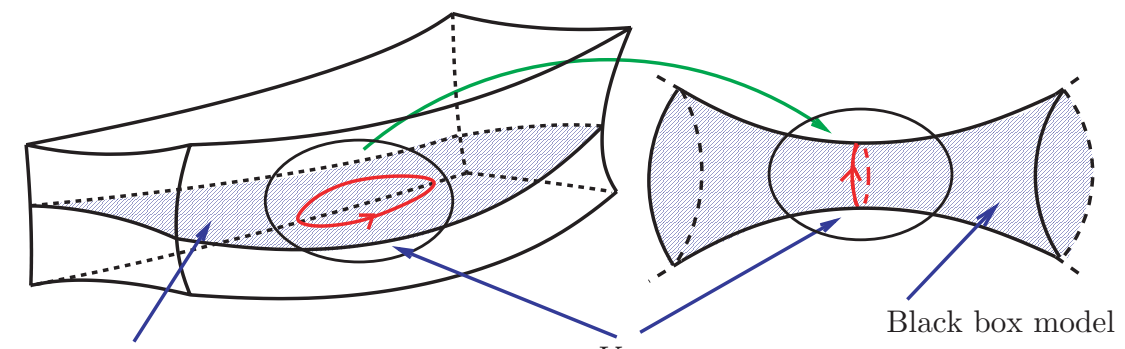

Energy surface $\{p=0\}$

Figure 3. A semi-classical black box with an hyperbolic trapped trajectory.

Suppose that $Q(h)$ is a family of bounded invertible operators on a Hilbert space $\mathcal{H}$. Suppose that there exist bounded operators

$$
\begin{aligned}
& U_{1}(h): L^{2}\left(X, \Omega_{X}^{\frac{1}{2}}\right) \longrightarrow \mathcal{H}, \\
& U_{2}(h): \mathcal{H} \longrightarrow L^{2}\left(X, \Omega_{X}^{\frac{1}{2}}\right), \\
& \chi^{\sharp}(h): \mathcal{H} \longrightarrow \mathcal{H},
\end{aligned}
$$

such that, microlocally near $V$, an open subset of $p^{-1}([-\delta, \delta])$, we have

$$
\begin{aligned}
& U_{2}(h) \circ U_{1}(h)=I d, \\
& U_{1}(h) \circ U_{2}(h)=\chi^{\sharp}(h), \\
& U_{1}(h) \circ P(h) \circ U_{2}(h)=Q(h) \circ \chi^{\sharp}(h) .
\end{aligned}
$$

In practice, the operators $U_{j}(h)$ are $h$-Fourier integral operators (see Proposition 2.1) but we do not need to make this assumption in the abstract presentation. Figure 3 shows our setup schematically in the case relevant for the proof of Theorem 2

Theorem 5. Let $P(h)$ and $Q(h)$ satisfy the assumptions above and let $V_{0}$ be an open relatively compact subset of $T^{*} X$. Suppose that $A \in \Psi_{h}^{0,0}\left(X, \Omega_{X}^{\frac{1}{2}}\right)$ is microlocally elliptic in $V_{0}$ and that there exists $T>0$ such that

$$
\begin{aligned}
& \forall \rho \in p^{-1}(0) \backslash V \exists 0<t<T, \epsilon \in\{ \pm 1\} \\
& \quad \exp \left(\epsilon s H_{p}\right)(\rho) \subset p^{-1}(0) \backslash V, 0<s<t, \quad \exp \left(\epsilon t H_{p}\right)(\rho) \in V_{0} .
\end{aligned}
$$


Suppose also that

$$
\left\|\chi^{\sharp}(h) Q(h)^{-1}\right\| \leq \frac{G(h)}{h}, \quad G(h) \geq 1 .
$$

Then for $u \in \mathcal{C}^{\infty}\left(X, \Omega_{X}^{\frac{1}{2}}\right)$ we have

$$
\|u\| \leq C \frac{G(h)}{h}\|P u\|+G(h)\|A u\| .
$$

We start with the following standard:

Lemma 4.1. Suppose that $p, A$, and $V$ satisfy (4.4). If $B \in \Psi^{0,0}\left(X, \Omega_{X}^{\frac{1}{2}}\right)$ and $W F(B) \subset T^{*} X \backslash V$, then

$$
\|B u\| \leq C h^{-1}\|P u\|+\|A u\|+\mathcal{O}\left(h^{\infty}\right)\|u\| .
$$

Proof. In view of the compactness of $p^{-1}(0)$ we can replace $V_{0}$ by a precompact neighbourhood of $V_{0} \cap p^{-1}(0)$. The assumption (4.4) then shows that it is enough to prove a local version of the estimate. We can suppose that $W F(A) \subset U$ where $U$ is a small neighbourhood of $m_{0} \in V_{0}$ and

$$
W F(B) \subset \bigcup_{0 \leq t \leq t_{0}} \exp \left(\epsilon t H_{p}\right)\left(U_{1}\right) \subset T^{*} X \backslash V, \quad U_{1} \Subset U .
$$

If $t_{0}$ is small enough, we can apply Proposition 2.2, as the estimate is clear in the case of $P=h D_{x_{1}}$. In general, we can then split the interval $\left[0, t_{0}\right]$ into subintervals in which the $t_{0}$-small argument can be applied.

Proof of Theorem [5. Suppose that $B_{1}$ satisfies

$$
W F\left(B_{1}\right) \subset V_{1}, \quad V \Subset V_{1}, \quad W F\left(I-B_{1}\right) \subset T^{*} X \backslash V .
$$

Then if $V_{1}$ is sufficiently close to $V$, using the second part of (4.3), we have

$$
\begin{aligned}
\left\|B_{1} u\right\| & =\left\|U_{2}\left(\chi^{\sharp}\right)^{2} U_{1} B_{1} u\right\|+\mathcal{O}\left(h^{\infty}\right)\|u\| \\
& =\left\|U_{2} \chi^{\sharp} Q^{-1} Q \chi^{\sharp} U_{1} B_{1} u\right\|+\mathcal{O}\left(h^{\infty}\right)\|u\| .
\end{aligned}
$$

If we now apply (4.5) and then (4.3) again, we obtain

$$
\begin{aligned}
\left\|B_{1} u\right\| & \leq C \frac{G(h)}{h}\left\|Q \chi^{\sharp} U_{1} B_{1} u\right\|_{\mathcal{H}}+\mathcal{O}\left(h^{\infty}\right)\|u\| \\
& \leq C \frac{G(h)}{h}\left(\|P u\|+\left\|\left[P, B_{1}\right] u\right\|\right)+\mathcal{O}\left(h^{\infty}\right)\|u\| \\
& \leq C \frac{G(h)}{h}\|P u\|+G(h)\left\|B_{2} u\right\|+\mathcal{O}\left(h^{\infty}\right)\|u\|,
\end{aligned}
$$

where $B_{2} \in \Psi^{0,0}\left(X, \Omega_{X}^{\frac{1}{2}}\right)$ satisfies

$$
W F\left(B_{2}\right) \subset V_{1} \backslash V, \quad W F\left(\left(I-B_{2}\right)\left[P, B_{1}\right]\right)=\emptyset .
$$

Lemma 4.1 now shows that

$$
\left\|B_{1} u\right\| \leq C \frac{G(h)}{h}\|P u\|+G(h)\|A u\|+\mathcal{O}\left(h^{\infty}\right)\|u\| .
$$

We now choose $B_{3} \in \Psi_{h}^{0,0}\left(X, \Omega_{X}^{\frac{1}{2}}\right)$ such that

$$
W F\left(B_{3}\right) \subset T^{*} X \backslash V \text { and } W F\left(I-B_{3}\right) \subset V_{1} .
$$

We can apply Lemma 4.1 with $B=B_{3}$ and that gives (4.6) as $\|u\| \simeq\left\|B_{1} u\right\|+$ $\left\|B_{3} u\right\|$. 
In some situations we can obtain improved estimates under a modified assumption on $Q^{-1}$. This modification will be crucial in Section [6] where we will prove (1.2). We present it separately not to obscure the simplicity of Theorem 5:

Theorem 5. Suppose that the assumptions of Theorem 5 hold and that in addition

$$
\left\|\chi^{\sharp}(h) Q^{-1} U_{1} \phi(h)\right\| \leq \frac{g(h)}{h},
$$

where $\phi(h)$ is a microlocal cut-off to a neighbourhood of $V_{1} \backslash V$, where $V_{1} \ni V$ is a small neighbourhood of $V$. Then we have,

$$
\|u\| \leq C \frac{G(h)}{h}\|P u\|+g(h)\|A u\| .
$$

Proof. We revisit the proof of Theorem 5. Instead of moving instantly to 4.9 from (4.8) using (4.6), we apply the identities (4.3) and write

$$
\begin{aligned}
\left\|B_{1} u\right\| & \leq C\left\|\chi^{\sharp} Q^{-1} Q \chi^{\sharp} U_{1} B_{1} u\right\|_{\mathcal{H}}+\mathcal{O}\left(h^{\infty}\right)\|u\| \\
& =\left\|\chi^{\sharp} Q^{-1} U_{1}\left(B_{1} P u+\left[P, B_{1}\right] u\right)\right\|_{\mathcal{H}}+\mathcal{O}\left(h^{\infty}\right)\|u\| \\
& \leq\left\|\chi^{\sharp} Q^{-1} U_{1} B_{1} P u\right\|_{\mathcal{H}}+\left\|\chi^{\sharp} Q^{-1} U_{1} \phi(h)\left[P, B_{1}\right] u\right\|_{\mathcal{H}}+\mathcal{O}\left(h^{\infty}\right)\|u\|,
\end{aligned}
$$

where we could insert the cut-off $\phi(h)$ due to the microsupport properties of $B_{1}$.

If we apply (4.6) and (4.10), we obtain a local version of (4.11):

$$
\left\|B_{1} u\right\| \leq C \frac{G(h)}{h}\|P u\|+\frac{g(h)}{h}\left\|\left[P, B_{1}\right] u\right\|+\mathcal{O}\left(h^{\infty}\right)\|u\| .
$$

The proof is then completed as in the case of Theorem 5 .

\section{Estimates in the homogeneous Case: Classical Control}

In this section we will adapt the semi-classical arguments of Section 4 to obtain a classical version of estimate (4.6). We start by modifying the black box assumptions where we essentially follow [37], 36] but change the ambient space from $\mathbb{R}^{n}$ to an arbitrary manifold.

Thus let $X$ be a compact $\mathcal{C}^{\infty}$ manifold with a (possibly empty) boundary $\partial X$. We consider an elliptic differential operator of order two,

$$
P_{0} \in \operatorname{Diff}^{2}\left(X, \Omega_{X}^{\frac{1}{2}}\right),
$$

with a domain $\mathcal{D}_{0} \subset L^{2}\left(X, \Omega_{X}^{\frac{1}{2}}\right)$. The choice of the domain includes the possible boundary conditions.

Let $Y \subset X$ be an open set. We also consider an auxiliary manifold $\widetilde{X}$, which coincides with $X$ on a neighbourhood $\tilde{Y}$ of $Y$; see Figure 4 for a visualization.

We then consider complex Hilbert spaces $\mathcal{H}, \mathcal{H}_{\text {bb }}$ with orthogonal decompositions

$$
\begin{aligned}
& \mathcal{H}=\mathcal{H}_{Y} \oplus L^{2}\left(X \backslash Y, \Omega_{X}^{\frac{1}{2}}\right), \\
& \mathcal{H}_{\mathrm{bb}}=\mathcal{H}_{Y} \oplus L^{2}\left(\widetilde{X} \backslash Y, \Omega_{\widetilde{X}}^{\frac{1}{2}}\right) .
\end{aligned}
$$

For $\mathcal{H}$ the orthogonal projections on the two factors are denoted by $\mathbb{1}_{Y}$ and $\mathbb{1}_{X \backslash Y}$, respectively. If $\chi_{j} \in \mathcal{C}^{\infty}(X)$ satisfy

$$
\operatorname{supp} \chi_{0} \subset \complement \operatorname{supp}\left(1-\chi_{1}\right) \subset \operatorname{supp} \chi_{1} \subset \tilde{Y}, \operatorname{supp}\left(1-\chi_{0}\right) \subset X \backslash \tilde{Y},
$$

then multiplication by $\chi_{j}$ is well defined on $\mathcal{H}$ and $\mathcal{H}_{\mathrm{bb}}$. 
On $L^{2}(X)$ and $\mathcal{H}_{\mathrm{bb}}$ we have unbounded operators $P_{0}$ and $P_{\mathrm{bb}}$, respectively, with domains

$$
\begin{aligned}
& \mathcal{D}_{0} \stackrel{\text { def }}{=} \mathcal{D}\left(P_{0}\right) \subset L^{2}\left(X, \Omega_{X}^{\frac{1}{2}}\right), \\
& \mathcal{D}_{\mathrm{bb}} \stackrel{\text { def }}{=} \mathcal{D}\left(P_{\mathrm{bb}}\right) \subset \mathcal{H}_{\mathrm{bb}} .
\end{aligned}
$$

A self-adjoint operator, $P: \mathcal{H} \longrightarrow \mathcal{H}$, has the domain $\mathcal{D} \subset \mathcal{H}$ satisfying the following conditions:

$$
\begin{gathered}
\mathbb{1}_{X \backslash Y} \mathcal{D}=\mathbb{1}_{X \backslash Y} \mathcal{D}_{0}, \mathbb{1}_{Y} \mathcal{D}=\mathbb{1}_{Y} \mathcal{D}_{\mathrm{bb}}, \\
\left(1-\chi_{1}\right) P=\left(1-\chi_{1}\right) P\left(1-\chi_{0}\right)=\left(1-\chi_{1}\right) P_{0}\left(1-\chi_{0}\right)=\left(1-\chi_{1}\right) P_{0}, \\
P \chi_{0}=\chi_{1} P \chi_{0}=\chi_{1} P_{\mathrm{bb}} \chi_{0}=P_{\mathrm{bb}} \chi_{0},
\end{gathered}
$$

for any functions satisfying (5.1). We use the notation from [37] and in particular we write

$$
\mathcal{D}^{\infty}=\bigcap_{k \in \mathbb{N}} \mathcal{D}\left(P^{k}\right), \quad \mathcal{D}^{-N}=\left(\mathcal{D}^{N}\right)^{*} .
$$

We also make another standard "black box" assumption:

$$
(P+i)^{-1} \text { is compact on } \mathcal{H} \text {. }
$$

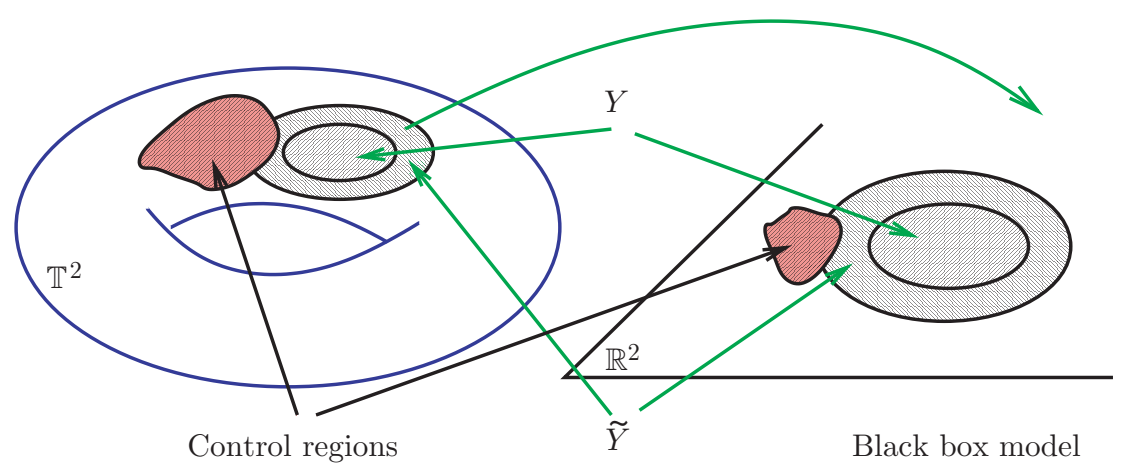

Figure 4 . The black box $Y$, its neighbourhood $\tilde{Y}$, in the case when $X=\mathbb{T}^{2}$ is the flat torus and $\widetilde{X}=\mathbb{R}^{2}$ is the plane.

As in previous sections we have two types of results. To obtain the assumptions of an analogue of Theorem 4, we need resolvent estimates based on black box resolvent estimates. That is provided in

Theorem 6. Suppose that $A: \mathcal{D}(A) \rightarrow \mathcal{H}_{1}, A_{\mathrm{bb}}: \mathcal{D}\left(A_{\mathrm{bb}}\right) \rightarrow \mathcal{H}_{1}$, where $\mathcal{H}_{1}$ is a Hilbert space, $\mathcal{D}(A) \supset \mathcal{D}^{\infty}, \mathcal{D}\left(A_{\mathrm{bb}}\right) \supset \mathcal{D}_{\mathrm{bb}}^{\infty}$, satisfy, for $u \in \mathcal{D}^{\infty}$ and $v \in \mathcal{D}_{\mathrm{bb}}^{\infty}$,

$$
\begin{gathered}
\left\|\mathbb{1}_{X \backslash Y} u\right\|_{\mathcal{H}} \leq C\langle\lambda\rangle^{-\frac{1}{2}}\|(P-\lambda) u\|_{\mathcal{H}}+\|A u\|_{\mathcal{H}_{1}}+\mathcal{O}\left(\langle\lambda\rangle^{-\infty}\right)\|u\|_{\mathcal{H}}, \\
\|v\|_{\mathcal{H}_{\mathrm{bb}}} \leq G(\lambda)\left(\langle\lambda\rangle^{-\frac{1}{2}}\left\|\left(P_{\mathrm{bb}}-\lambda\right) v\right\|_{\mathcal{H}_{\mathrm{bb}}}+\left\|A_{\mathrm{bb}} v\right\|_{\mathcal{H}_{1}}\right), \quad|\lambda| \rightarrow \infty, \\
A \chi_{0}=\chi_{1} A \chi_{0}=\chi_{1} A_{\mathrm{bb}} \chi_{0}=A_{\mathrm{bb}} \chi_{0}, \quad G(\lambda) \geq 1, \\
\forall k \exists C_{k},\left\|A_{b b} \chi_{0} u\right\| \leq C_{k}\left(\|A u\|_{\mathcal{H}_{1}}+\|u\|_{\mathcal{D}^{-k}}\right)
\end{gathered}
$$

for any $\chi_{j}$ 's satisfying (5.1). Then $\exists \lambda_{0}>0 ; \forall \lambda>\lambda_{0}$,

$$
\|u\|_{\mathcal{H}} \leq C_{1} G(\lambda)\left(\langle\lambda\rangle^{-1 / 2}\|(P-\lambda) u\|_{\mathcal{H}}+\|A u\|_{\mathcal{H}_{1}}\right)
$$


Proof. We first prove the following estimate:

$$
\langle\lambda\rangle^{-1 / 2}\left\|\left[P_{0}, \chi_{0}\right] u\right\|_{\mathcal{H}} \leq C\left(\langle\lambda\rangle^{-1 / 2}\|(P-\lambda) u\|_{\mathcal{H}}+\left\|u \mathbb{1}_{X \backslash Y}\right\|_{\mathcal{H}}\right) .
$$

Indeed, the ellipticity of $P_{0}$ gives

$$
\begin{aligned}
\|u\|_{H^{2}\left(\operatorname{supp}\left(\nabla \chi_{0}\right)\right)} & \leq C\left(\left\|P_{0} u\right\|_{L^{2}(X \backslash Y)}+\|u\|_{L^{2}(X \backslash Y)}\right) \\
& \leq C\left(\|(P-\lambda) u\|_{L^{2}(X \backslash Y)}+\langle\lambda\rangle\|u\|_{L^{2}(X \backslash Y)}\right) .
\end{aligned}
$$

Using the inequality $\|u\|_{H^{1}} \leq C \sqrt{\|u\|_{H^{2}}\|u\|_{L^{2}}}$, we get (5.4).

We now turn to the proof of (5.3). The black box assumptions give

$$
\left(P_{\mathrm{bb}}-\lambda\right) \chi_{0} u=(P-\lambda) \chi_{0} u=\left[P_{0}, \chi_{0}\right] u+\chi_{0}(P-\lambda) u .
$$

Using (5.2), we obtain

$$
\begin{aligned}
\left\|\chi_{0} u\right\|_{\mathcal{H}} & =\left\|\chi_{0} u\right\|_{\mathcal{H}_{\mathrm{bb}}} \\
& \leq G(\lambda)\left(\langle\lambda\rangle^{-1 / 2}\left(\left\|\chi_{0}(P-\lambda) u\right\|_{\mathcal{H}_{\mathrm{bb}}}+\left\|\left[P_{0}, \chi_{0}\right] u\right\|_{\mathcal{H}_{\mathrm{bb}}}\right)+\left\|A_{\mathrm{bb}} \chi_{0} u\right\|_{\mathcal{H}_{1}}\right) .
\end{aligned}
$$

Above, we can replace the norms in $\mathcal{H}_{\mathrm{bb}}$ by norms in $\mathcal{H}$ and, using (5.2) and (5.4), this implies

$$
\left\|\chi_{0} u\right\|_{\mathcal{H}} \leq C G(\lambda)\left(\langle\lambda\rangle^{-1 / 2}\|(P-\lambda) u\|_{\mathcal{H}}+\|A u\|_{\mathcal{H}_{1}}+\mathcal{O}\left(\langle\lambda\rangle^{-\infty}\right)\|u\|_{\mathcal{H}}+\|u\|_{\mathcal{D}^{-k}}\right) .
$$

To conclude the proof, we use the first inequality in (5.2) and the fact that

$$
\|u\|_{\mathcal{D}^{-k}}=\left\|(P+i)^{-k} u\right\|_{\mathcal{H}} \leq C_{k}\left(\langle\lambda\rangle^{-k}\|u\|_{\mathcal{H}}+\|(P-\lambda) u\|_{\mathcal{H}}\right) .
$$

Remark 1. In the proof above, the operators $A$ and $A_{\mathrm{bb}}$ could depend on $\lambda$ as long as the assumptions are uniform in $\lambda$.

The difference between the semi-classical and classical control estimates, (3.3) and (5.9) below, is more serious. In the classical case the low energy contribution does not allow an explicit time dependent constant as we have in (3.3) (compare (5.9) and (5.26) below). As investigated recently in [33, violent behaviour is expected when fast control is a goal.

Theorem 7. Suppose that $A: \mathcal{D}(A) \rightarrow \mathcal{H}_{1}$, where $\mathcal{H}_{1}$ is a Hilbert space, $\mathcal{D}(A) \supset$ $\mathcal{D}^{\infty}$, satisfies the following condition: for all $N$ there exists $C_{N}$ such that for all $k \in \mathbb{N}$ and $u \in \mathcal{D}^{\infty}$,

$$
\begin{gathered}
\left\|A \psi\left(2^{-k} P\right) u\right\|_{\mathcal{H}_{1}}+\left\|A(1-\psi)\left(2^{-k} P\right) u\right\|_{\mathcal{H}_{1}} \\
\leq C_{N}\left(\|A u\|_{\mathcal{H}_{1}}+2^{-k N}\|u\|_{\mathcal{D}^{-N}}\right)
\end{gathered}
$$

$\psi \in C_{0}^{\infty}((1 / 2,2))$.

Suppose also that for all $\lambda \in \mathbb{R}$ and $u \in \mathcal{D}^{\infty}$ we have

$$
\|u\|_{\mathcal{H}} \leq G(\lambda)\|(P-\lambda) u\|_{\mathcal{H}}+g(\lambda)\|A u\|_{\mathcal{H}_{1}},
$$

where $G$ and $g$ satisfy

(5.8)

$\langle\lambda\rangle^{-1} \leq G(\lambda) \leq\langle\lambda\rangle^{N_{0}}, \quad C\langle\lambda\rangle^{-N_{0}} \leq g(\lambda) \leq C^{\prime}\langle\lambda\rangle^{N_{0}}, \quad g(\lambda / 2) \leq C g(\lambda) \leq C^{\prime} g(2 \lambda)$.

We also assume the following weak continuity property (see Remark 0 for a discussion). There exist $N_{1} \in \mathbb{N}$ and a Hilbert space $\mathcal{H}^{\sharp}$ such that $\mathcal{H}_{1} \subset \mathcal{H}^{\sharp}$ continuously, and the operator $A e^{i t P}$ is continuous from $\mathcal{D}^{-N_{0}}$ to $H_{\text {loc }}^{-N_{1}}\left(\mathbb{R}_{t}, \mathcal{H}^{\sharp}\right)$. 
Then there exist constants $C_{0}$ and $C_{1}=C_{1}(T)$ such that for any

$$
T>C_{1} \limsup _{|\lambda| \rightarrow \infty} G(\lambda)
$$

we have for $u \in \mathcal{D}^{\infty}$,

$$
\left\|\langle g(P)\rangle^{-1} u\right\|_{\mathcal{H}}^{2} \leq C_{1}(T) \int_{0}^{T}\left\|e^{i t P} A u\right\|_{\mathcal{H}_{1}}^{2} d t .
$$

Remark 2. In the case where the operator $P$ is the Laplace operator with Dirichlet boundary conditions, the weak continuity is satisfied in the two following typical situations:

(1) If $A$ is a pseudodifferential operator supported in the interior of $X$, then $\mathcal{H}_{1}=L^{2}(X)$ and we can take $\mathcal{H}^{\sharp}$ to be another Sobolev space $H^{-s}(X)$.

(2) If $A u=\partial_{\nu} \uparrow_{\Gamma}$ where $\Gamma \subset \partial \Omega$ and $\partial_{\nu}$ is the normal derivative to the boundary, then we can take $\mathcal{H}^{\sharp}=\mathcal{H}_{1}=L^{2}(\partial X)$ as standard trace regularity results for solutions of Schrödinger equations show that the assumptions hold with $N_{0}$ sufficiently large.

Proof of Theorem 7 . We follow closely the proof of Theorem 4 observing first that, with $\Psi \in C_{0}^{\infty}(] 1 / 2,2[)$ equal to 1 close to 1 , (5.7) and (5.6) imply

$$
\begin{aligned}
& \langle g(\lambda)\rangle^{-1}\|\Psi(P /\langle\lambda\rangle) u\|_{\mathcal{H}} \\
& \quad \leq G(\lambda)\langle g(\lambda)\rangle^{-1}\|\Psi(P /\langle\lambda\rangle)(P-\lambda) u\|_{\mathcal{H}}+C_{N}\|A u\|_{\mathcal{H}_{1}}+C\langle\lambda\rangle^{-N}\|u\|_{\mathcal{D}^{-N}}
\end{aligned}
$$

which in turn implies

$$
\begin{aligned}
& \left\|\langle g(P)\rangle^{-1} \Psi(P /\langle\lambda\rangle) u\right\|_{\mathcal{H}} \\
& \quad \leq G(\lambda)\left\|\langle g(P)\rangle^{-1}(P-\lambda) u\right\|_{\mathcal{H}}+C_{N}\|A u\|_{\mathcal{H}_{1}}+C\langle\lambda\rangle^{-N}\|u\|_{\mathcal{D}^{-N}} .
\end{aligned}
$$

The functional calculus of self adjoint operators gives

$$
\begin{aligned}
\left\|(1-\Psi)(P /\langle\lambda\rangle)\langle g(P)\rangle^{-1} u\right\|_{\mathcal{H}} & \leq \sup _{\xi}\left|\frac{(1-\Psi)(\xi /\langle\lambda\rangle)}{\xi-\lambda}\right|\left\|\langle g(P)\rangle^{-1} u\right\|_{\mathcal{H}} \\
& \leq \frac{C}{1+|\lambda|}\left\|\langle g(P)\rangle^{-1} u\right\|_{\mathcal{H}},
\end{aligned}
$$

which, using (5.6) again, and (5.8) implies (taking $N$ large enough) that for $|\lambda|$ large enough,

$$
\left\|\langle g(P)\rangle^{-1} u\right\|_{\mathcal{H}} \leq C G(\lambda)\left\|\langle g(P)\rangle^{-1}(P-\lambda) u\right\|_{\mathcal{H}}+C\|A u\|_{\mathcal{H}_{1}} .
$$

Proceeding as in the proof of Theorem 4 we define $v(t)=\exp (i t P) u$. We introduce a function $\psi \in C_{0}^{\infty}(] 0,1[)$ and put

$$
w(t)=\psi\left(\frac{t}{T}\right) v(t),
$$

so that

$$
\left(i \partial_{t}-P\right) w(t)=\frac{i}{T} \psi^{\prime}\left(\frac{t}{T}\right) v(t) .
$$

Because of the compact support we can take the Fourier transform in $t$ which gives

$$
(\tau-P) \widehat{w}(\tau)=\frac{i}{T} \mathcal{F}_{t \rightarrow \tau}\left(\psi^{\prime}(\bullet / T) v\right)(\tau) .
$$


Let $\varrho$ be a large constant to be fixed later. For $\langle\tau\rangle \geq \varrho / 2$ we estimate $\widehat{w}(\tau)$ using (5.12) which gives

$$
\left\|\langle g(P)\rangle^{-1} \widehat{w}(\tau)\right\|_{\mathcal{H}} \leq C \frac{G(\tau)}{T}\left\|\langle g(P)\rangle^{-1} \mathcal{F}_{t \rightarrow \tau}\left(\psi^{\prime}(\bullet / T) v\right)(\tau)\right\|_{\mathcal{H}}+C\|A \widehat{w}(\tau)\|_{\mathcal{H}_{1}} .
$$

For $\langle\tau\rangle \leq \varrho / 2$ we simply write, with $\chi \in C_{0}^{\infty}(]-1,1[)$ equal to 1 on $[-1 / 2,1 / 2]$,

$$
\widehat{w}(\tau)=\int_{t \in \mathbb{R}} e^{i t(P-\tau)} \psi\left(\frac{t}{T}\right)(\chi(P / \varrho) u+(1-\chi(P / \varrho)) u) d t .
$$

The contribution of the first term is bounded (in $\mathcal{H})$ by $\|\chi(P / \varrho) u\|_{\mathcal{H}}$ and by integrations by parts with the operator $\frac{i \partial_{t}}{P-\tau}$ we can bound the contribution of the second term by

$$
C_{N}\left\|\frac{1}{(1+|T|+|\varrho|+\langle P\rangle)^{N}} u\right\|_{\mathcal{H}} .
$$

From (5.13), (5.14), (5.15) and the bounds on the weight $g$, we get

$$
\begin{aligned}
\left\|\langle g(P)\rangle^{-1} \widehat{w}(\tau)\right\|_{L^{2}\left(\mathbb{R}_{\tau}, \mathcal{H}\right)}^{2} & \\
\leq & C\left(\frac{\sup _{|\tau| \geq \rho / 2} G(\tau)}{T}\right)^{2}\left\|\langle g(P)\rangle^{-1} \mathcal{F}_{t \rightarrow \tau}\left(\psi^{\prime}(\bullet / T) v\right)\right\|_{L^{2}\left(\mathbb{R}_{\tau}, \mathcal{H}\right)}^{2} \\
& +C \int_{0}^{T}\left\|A e^{i t P} u\right\|_{\mathcal{H}_{1}}^{2} d t+C_{\varrho}\left\|\mathbb{1}_{\langle P\rangle \leq \rho} u\right\|_{\mathcal{H}}^{2}+C\langle\varrho\rangle^{-N_{0}}\|u\|_{\mathcal{D}^{-N_{0}}}^{2} .
\end{aligned}
$$

Note that

$$
\left\|\langle g(P)\rangle^{-1} \widehat{w}(\tau)\right\|_{L^{2}\left(\mathbb{R}_{\tau}, \mathcal{H}\right)}=T^{1 / 2}\|\Psi\|_{L^{2}}\left\|\langle g(P)\rangle^{-1} u\right\|_{\mathcal{H}}
$$

and

$$
\left\|\langle g(P)\rangle^{-1} \mathcal{F}_{t \rightarrow \tau}\left(\psi^{\prime}(\bullet / T) v\right)\right\|_{L^{2}\left(\mathbb{R}_{\tau}, \mathcal{H}\right)}=T^{1 / 2}\left\|\Psi^{\prime}\right\|_{L^{2}}\left\|\langle g(P)\rangle^{-1} u\right\|_{\mathcal{H}} .
$$

Consequently, taking $\rho$ large enough, the assumption $T>C_{1} \lim \sup _{|\lambda| \rightarrow \infty} G(\lambda)$ ensures that we can eliminate the first and the last terms in the right-hand side and get

$$
\left\|\langle g(P)\rangle^{-1} u\right\|_{\mathcal{H}} \leq C \int_{0}^{T}\left\|A e^{i t P} u\right\|_{\mathcal{H}_{1}}^{2} d t+C_{\varrho}\left\|\mathbb{1}_{\langle P\rangle \leq \rho} u\right\|_{\mathcal{H}}^{2} .
$$

To eliminate the last term, we use the compactness-uniqueness argument from [2] which we now recall. Proceeding by contradiction, we obtain a sequence $\left(u_{n}\right)$ such that

$$
C\left\|\mathbb{1}_{\langle P\rangle \leq \rho} u_{n}\right\|_{\mathcal{H}}^{2} \geq 1=\left\|\langle g(P)\rangle^{-1} u_{n}\right\|_{\mathcal{H}}^{2} \geq n \int_{0}^{T}\left\|A e^{i t P} u_{n}\right\|_{\mathcal{H}_{1}}^{2} d t .
$$

Define

$$
H_{T}=\left\{u \in \mathcal{D}^{-N_{0}}:\left\|\langle g(P)\rangle^{-1} u\right\|_{\mathcal{H}}^{2}+\int_{0}^{T}\left\|A e^{i t P} u_{n}\right\|_{\mathcal{H}_{1}}^{2} d t<+\infty\right\}
$$

with its natural norm (the definition makes sense because of the weak continuity property of $\left.A e^{i t P}\right)$. Due to the assumption (5.8) and the weak continuity property of $A e^{i t P}, H_{T}$ is a Hilbert space which is continuously embedded in $\mathcal{D}^{-N_{0}}$. The sequence $\left(u_{n}\right)$ is bounded in $H$ and we can extract a subsequence converging weakly 
in $H$ to a limit $u$. Using the compactness of $(P+i)^{-1}$, the operator $\mathbb{1}_{\langle P\rangle \leq \rho}$ is also compact on $\mathcal{D}^{-N_{0}}$. By passing to the limit, we see that $u$ satisfies

$$
C\left\|\mathbb{1}_{\langle P\rangle \leq \rho} u\right\|_{\mathcal{H}}^{2} \geq 1
$$

and

$$
0=\int_{0}^{T}\left\|A e^{i t P} u\right\|_{\mathcal{H}_{1}}^{2} d t
$$

The contradiction comes from the following:

Lemma 5.1. Let

$$
N=\left\{u \in H_{T}: 0=\int_{0}^{T}\left\|A e^{i t P} u\right\|_{\mathcal{H}_{1}}^{2} d t\right\} .
$$

Then $N=\{0\}$.

Proof. We first show that $N$ is invariant under the action of the operator $P$. Using that $P e^{i t P} u=i \partial_{t} e^{i t P} u$, the only thing to show is that if $u \in N$, then $\left\|\langle g(P)\rangle^{-1} P u\right\|_{\mathcal{H}}$ is bounded.

We put $v(t)=e^{i t P} u$ and apply (5.19) with $T$ replaced by $T-\varepsilon_{0}$ to the sequence of functions

$$
v_{\varepsilon}=i \frac{v(t+\varepsilon)-v(t)}{\varepsilon} .
$$

We get for $0<\varepsilon<\varepsilon_{0}$

$$
\left\|\left.\langle g(P)\rangle^{-1} v_{\varepsilon}\right|_{t=0}\right\|_{\mathcal{H}} \leq C\left\|\left.\mathbb{1}_{\langle P\rangle \leq \rho} v_{\varepsilon}\right|_{t=0}\right\|_{\mathcal{H}}^{2}
$$

and using that $\left.v_{\varepsilon}\right|_{t=0}$ converges to $\left.i \partial_{t} u\right|_{t=0}=\left.P u\right|_{t=0}$ in $\mathcal{D}^{-N_{0}-1}$, we obtain that the right-hand side is bounded as $\varepsilon$ tends to 0 . Consequently, we can extract a subsequence $v_{\varepsilon}$ converging in $H_{T-\varepsilon_{0}}$. The limit is necessarily (due to the weak continuity property) $P u$ which implies that $P u \in N$. To conclude, remark that $\left\|\mathbb{1}_{\langle P\rangle \leq \rho} u_{n}\right\|_{\mathcal{H}}^{2}$ is a norm on $N$ equivalent to the natural norm. Consequently $N$ is finite dimensional. The space $N$ is invariant by the operator $P$ which consequently has an eigenvector. But any eigenvector of $P$ in $N$ satisfies $A u=0$ and is equal to 0 due to (5.7). Consequently $N=\{0\}$.

\section{EXAMPLES AND APPLICATIONS}

In this section we present several applications of our method, giving, in particular, the proof of Theorems 1, 2 and 3 stated in the introduction.

6.1. Geometric control. As in the introduction we consider $\Omega$, a smooth domain in $\mathbb{R}^{d}, \Gamma \subset \partial \Omega$, and we fix $T>0$. For any $g \in L^{2}([0, T] \times \Gamma)$, we consider $u$ the solution of the mixed problem (1.1). The goal is to find conditions on $\Gamma$ so that there exists a large class of functions $u_{0}$ which can be "controlled" by $g$, in the sense that

$$
u \uparrow_{t=T}=0 .
$$

When one deals with such control problems, the usual first step (called the H.U.M. method) is a dual approach: For any $g \in L^{2}([0, T] \times \Gamma)$, consider $v$, the 
solution of the mixed problem

$$
\begin{aligned}
\left(i \partial_{t}-\Delta\right) v & =0, \\
v \uparrow_{\partial \Omega} & =g \mathbb{1}_{[0, T] \times \Gamma}, \\
\left.v\right|_{t=T} & =0,
\end{aligned}
$$

and define $S(g)=v \uparrow_{t=0}$. In this setting the control problem is to determine the range of the operator $S$. For that consider $u=e^{i t \Delta_{D}} u_{0}$, the solution of

$$
\begin{aligned}
\left(i \partial_{t}-\Delta\right) u & =0, \\
u \uparrow_{\partial \Omega} & =0, \\
u \uparrow_{t=0} & =u_{0} .
\end{aligned}
$$

We then define a new operator

$$
T\left(u_{0}\right) \stackrel{\text { def }}{=} \partial_{\nu} u \uparrow_{[0, T] \times \Gamma} .
$$

Lemma 6.1 (H.U.M. method, [30]). The operators $S$ and $T$ have the continuity properties

$$
\begin{aligned}
& S: L^{2}([0, T] \times \Gamma) \longrightarrow H^{-1}(\Omega), \\
& T: H_{0}^{1}(\Omega) \longrightarrow L^{2}([0, T] \times \Gamma),
\end{aligned}
$$

and the adjoint of $T$ is equal to $i S$.

Proof. Let us define $X(t, x)=\phi(t) Y(x)$, where $Y(x)$ is a smooth vector field on $\bar{\Omega}$ equal to $\partial_{n}$ close to $\partial \Omega$ and $\phi \in \mathcal{C}_{\mathrm{c}}^{\infty}(\mathbb{R})$. With $u(t, x)$ as in (6.2), an application of Stokes's formula gives

$$
\int_{\mathbb{R}_{t} \times \Omega}\left[\left(i \partial_{t}-\Delta\right), X\right] u \bar{u} d x d t=-\int_{\mathbb{R}_{t} \times \partial \Omega}(X u)\left\lceil_{\partial \Omega} \overline{\partial_{\nu} u} d \sigma d t .\right.
$$

Since the operator $\left[\left(i \partial_{t}-\Delta\right), X\right]$ is a second order differential operator in the $x$ variable only, the left-hand side in (6.4) is bounded by $C\|u\|_{L^{\infty}\left(\mathbb{R}_{t}, H_{0}^{1}(\Omega)\right)}^{2} \sim\left\|u_{0}\right\|_{H_{0}^{1}(\Omega)}^{2}$. The right-hand side is equal to

$$
\left\|\phi(t) \partial_{\nu} u\right\|_{L^{2}\left(\mathbb{R}_{t} \times \partial \Omega\right)}^{2}
$$

and that implies the continuity of the operator $T$.

To see the duality of $T$ and $i S$, we multiply (1.1) by $\bar{u}$ and integrate over $[0, T] \times \Omega$. Using the boundary conditions satisfied by $u$ and $v$, we obtain

$$
\begin{aligned}
0 & =\int_{0}^{T} \int_{\Omega}\left(i \partial_{t}-\Delta\right) v \bar{u} d x d t \\
& =i\left[\int_{\Omega} v \bar{u}\right]_{0}^{T}+\int_{0}^{T} \int_{\partial \Omega}\left(-\partial_{\nu} v \bar{u}+v \overline{\partial_{\nu} u}\right) d \sigma d t \\
& =-i \int_{\Omega} S(g) \overline{u_{0}}+\int_{0}^{T} \int_{\Gamma} g \overline{T\left(u_{0}\right)} d \sigma d t
\end{aligned}
$$

which proves the claim $T^{*}=i S$. The continuity of the operator $S$ follows.

As an application of Lemma 6.1 we see that the range of the operator $S$ is dense (approximate controllability) if and only if the operator $T$ is injective. Also, the range of $S$ is equal to $H^{-1}(\Omega)$ (exact controllability) if and only if

$$
\exists C>0 \quad\left\|T\left(u_{0}\right)\right\|_{L^{2}([0, T] \times \Gamma)} \geq C\left\|u_{0}\right\|_{H_{0}^{1}(\Omega)} .
$$


Finally, any reasonable estimate implying the injectivity of the operator $T$ will give information on the range of $S$. For example

$$
\left\|T\left(u_{0}\right)\right\|_{L^{2}([0, T] \times \Gamma)} \geq C\left\|u_{0}\right\|_{H^{-s}(\Omega)} \Longrightarrow H^{s}(\Omega) \cap H_{0}^{1}(\Omega) \subset \operatorname{Range}(S) .
$$

In the context of exact geometric control, the basic result was obtained by Lebeau [29] (see also [30] and [44]). It involves the natural concepts of the broken geodesic flow and of nondiffractive points (see [32] and also [6]):

Theorem 8. Suppose that $\Gamma$ controls $\Omega$ geometrically, that is, (6.6)

$\exists L_{0}$ such that every trajectory of length $L_{0}$ meets $\Gamma$ at a nondiffractive point, where trajectories are with respect to the broken geodesic flow. Then for any $T>0$ and any $u_{1} \in H^{-1}(\Omega)$ there exists $g \in L^{2}([0, T] \times \Gamma)$ such that $\left.S(g)\right|_{t>T} \equiv 0$.

Proof. We first recall that as an application of Lions's H.U.M. method (Lemma6.1), we see that Theorem 8 is equivalent to

$$
\exists C>0 ; \quad\left\|u_{0}\right\|_{H_{0}^{1}(\Omega)} \leq C \| \partial_{\nu}\left(e^{i t \Delta_{D}} u_{0}\right)\left\lceil_{[0, T] \times \Gamma} \|_{L^{2}([0, T] \times \Gamma)} .\right.
$$

Estimate (6.7) follows from Theorem 7 and the following resolvent estimate:

$$
\begin{array}{r}
\exists C>0 \forall z \in \mathbb{C} \backslash \mathbb{R},\|R(z) f\|_{H^{1}(\Omega)}+\sqrt{|z|}\|R(z) f\|_{L^{2}(\Omega)} \\
\leq C\left\|\partial_{\nu} R(z) f\right\|_{L^{2}(\Gamma)}+C\|f\|_{L^{2}(\Omega)},
\end{array}
$$

where $R(z)=\left(-\Delta_{D}-z\right)^{-1}$, with $\Delta_{D}$ the Dirichlet Laplacian on $\Omega$. In fact, we can simply put $A u=\partial_{\nu} u \Gamma_{\Gamma}$ and $\mathcal{H}_{1}=L^{2}(\Gamma)$. To establish (6.8), we remark first that due to standard elliptic estimates, the only problem is when $z$ is close to the spectrum of $-\Delta, \mathbb{R}^{+}$. We can use the microlocal defect measures arguments as in [6]: we first prove (6.8) for large $z$ and argue by contradiction. We obtain sequences $z_{n}$ such that $\operatorname{Re} z_{n} \rightarrow+\infty$ and $\operatorname{Im} z_{n} \rightarrow 0$ and with $u_{n}$ a solution of

$$
\begin{gathered}
\left(-\Delta-z_{n}\right) u_{n}=f_{n}, \quad\left\|u_{n}\right\|_{L^{2}(\Omega)}+\frac{1}{\sqrt{z_{n}}}\left\|\nabla_{x} u_{n}\right\|_{L^{2}(\Omega)}=1, \\
\left\|f_{n}\right\|_{L^{2}(\Omega)}=o\left(\sqrt{\operatorname{Re} z_{n}}\right), \\
\left\|\partial_{n} u_{n}\right\|_{L^{2}(\Gamma)}=o\left(\sqrt{\operatorname{Re} z_{n}}\right) .
\end{gathered}
$$

Denote $h_{n}=\left(\operatorname{Re}\left(z_{n}\right)\right)^{-1 / 2}$. Then, modulo the extraction of a subsequence (see [19] 6]), there exists a positive Radon measure (a semi-classical defect measure on $T^{*} \mathbb{R}^{d}$ ) such that, if $u_{n}$ is the extension of $u_{n}$ by 0 outside of $\bar{\Omega}$, we have

(1) For any $h$-pseudodifferential operator, $A$, on $\mathbb{R}^{d}$,

$$
\left\langle\mu, \sigma_{0}(A)\right\rangle=\lim _{n \rightarrow+\infty}\left(A\left(x, h_{n} D_{x}\right) \underline{u_{n}}, \underline{u_{n}}\right)_{L^{2}\left(\mathbb{R}^{d}\right)} .
$$

(2) The measure $\mu$ is supported in the semi-classical characteristic variety:

$$
\operatorname{supp}(\mu) \subset T^{*} \mathbb{R}^{d} \cap\left\{(x, \xi) ; x \in \bar{M},|\xi|^{2}=1\right\} .
$$

Furthermore (see [6, 9]), using (6.10), we obtain that this measure is invariant along the generalized bicharacteristic flow. In the interior, this property is straightforward, whereas, near the boundary, it is more involved. In particular, we can show that the measure of the hyperbolic set (corresponding to transversal reflections) is equal to 0 . This allows a definition of a bicharacteristic flow on the set (6.12), $\mu$ almost everywhere. Due to (6.11) the measure is equal to 0 near any nondiffractive 
point in $\Gamma$ (see [8]), which, by (6.6), implies that the measure is identically null. Finally the contradiction arises from the fact that according to (6.9) the measure has total mass 1 .

The proof of 6.8 for $z \leq C$ is obtained by a contradiction argument (and compactness) and the classical uniqueness theorem for second order elliptic operators (for this point we simply use that $\stackrel{\circ}{\Gamma} \neq \emptyset$ ).

6.2. Ikawa's black box. In the proof of Lebeau's theorem we did not use any "black-box" technology. As illustrated by Figure 1, we can employ it in

Proof of Theorem 1. As in the proof of Theorem 8 we use the H.U.M. method and Theorem 7 to reduce the argument to the estimate

$$
\|R(z) f\|_{H^{1}(\Omega)}+\sqrt{|z|}\|R(z) f\|_{L^{2}(\Omega)} \leq C \log (|z|)\left(\left\|\partial_{n} R(z) f\right\|_{L^{2}(\Gamma)}+\|f\|_{L^{2}(\Omega)}\right),
$$

for $\operatorname{Im} z \neq 0$. This estimate follows from Theorem 6 and the following consequence of the work of Ikawa [26, Theorem 2.1]. Suppose that $R_{\mathrm{bb}}(k)$ is the outgoin 1 resolvent for the Dirichlet problem in the exterior of the union of convex obstacles satisfying

- (convhull $\left.\Theta_{j} \cup \Theta_{k}\right) \cap \Theta_{l}=\emptyset, \quad j \neq l \neq k$.

- Denote by $\kappa$ the infimum of the principal curvatures of the boundaries of the obstacles $\Theta_{i}$ and by $L$ the infimum of the distances between two obstacles. Then if $N>2$, we assume that $\kappa L>N$ (no assumption if $N=2$ ).

Then there exist $\alpha>0, C_{0}$, and $N_{0}$ such that for $\operatorname{Im} k>-\alpha$ we have

$$
\left\|\chi R_{\mathrm{bb}}(k) \chi\right\|_{L^{2} \rightarrow L^{2}} \leq C_{0}\langle k\rangle^{N_{0}}, \quad \chi \in \mathcal{C}_{c}^{\infty}\left(\mathbb{R}^{n}\right) .
$$

An application of the maximum principle as in [40, Lemma 2] and [7, Lemma 4.10] (see also Lemma A.2 below) gives a bound for $k \in \mathbb{R}$

$$
\left\|\chi R_{\mathrm{bb}}(k) \chi\right\|_{L^{2} \rightarrow L^{2}} \leq C_{1} \frac{\log \langle k\rangle}{\langle k\rangle},
$$

and that gives the "black-box" assumption (5.2) with $G(\lambda)=\log \langle\lambda\rangle$ and $A_{\mathrm{bb}} \equiv$ 0 .

6.3. Bunimovich stadium with the flat part as the black box. Our next control theoretical application is a new result about high frequency scarring in the case of the Bunimovich stadium2. The same argument applies also in recent examples related to quantum unique ergodicity [16], [42] where the flat part "black box" needs to be replaced by a flat torus. The result which we use in the black box (see Proposition 6.1 below) applies to that case as well.

Theorem 3]. Consider $\Omega$ the Bunimovich stadium associated to a rectangle $R$ and $\omega \subset \Omega$ which controls a neighbourhood of $\Omega \backslash R$ geometrically. For any solution of the equation $(\Delta-z) v=f, u \uparrow_{\partial \Omega}=0$, we then have

$$
\|v\|_{L^{2}(\Omega)} \leq C\left(\|f\|_{L^{2}(\Omega)}+\left\|\mathbb{1}_{\omega} v\right\|_{L^{2}(\omega)}\right) .
$$

\footnotetext{
${ }^{1}$ The outgoing resolvent is the meromorphic continuation of $\left(-\Delta-k^{2}\right)^{-1}$ from $\operatorname{Im} k>0$

${ }^{2}$ Which is perhaps the most celebrated example of a convex chaotic billiard.
} 


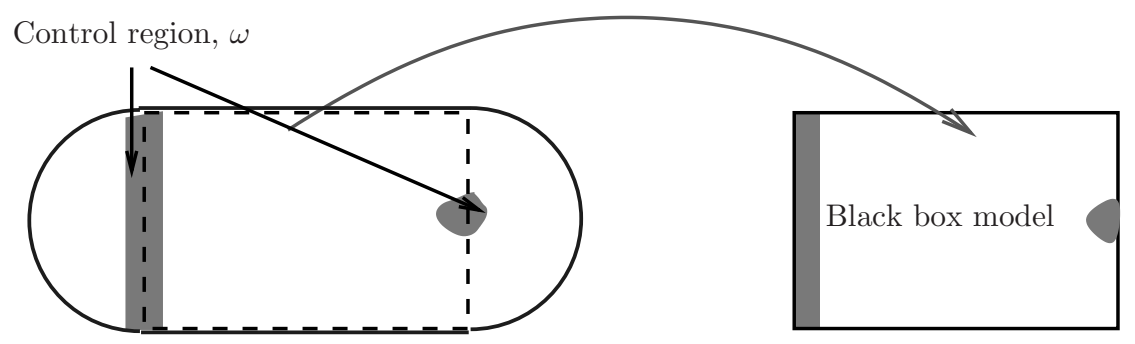

Figure 5. Control on the Bunimovich stadium.

Theorem 3 follows by considering the case of $f \equiv 0$. We also have the following immediate consequence of Theorem 7 .

Theorem 9. Consider $\Omega$ the Bunimovich stadium associated to a rectangle $R$ and $\omega \subset \Omega$ which controls $\Omega \backslash R$ geometrically. Then there exist $T>0$ and $C>0$ such that

$$
\left\|u_{0}\right\|_{L^{2}(\Omega)}^{2} \leq C \int_{0}^{T}\left\|\mathbb{1}_{\omega} e^{i t \Delta} u_{0}\right\|_{L^{2}(\Omega)}^{2} .
$$

In fact, by using a temporal black box, we could prove Theorem 9 for any $T>0$.

Before presenting the proof (see also [10] for a self-contained and elementary proof of a slightly weaker result), we briefly discuss the significance of the result. The Schnirelman theorem for the case of bounded planar domains (proved in [19] and [43]) implies that

$$
\begin{gathered}
\exists\left\{n_{k}\right\}_{k=1}^{\infty} \subset \mathbb{N} \quad \lim _{N \rightarrow \infty} \frac{1}{N}\left|\left\{n_{k}: k \leq N\right\}\right|=1, \quad \int_{V}\left|u_{n_{k}}(x)\right|^{2} d x \longrightarrow \operatorname{Area}(V), \\
V \subset \Omega, \quad-\Delta u_{\ell}=\lambda_{\ell} u_{\ell}, \quad \int_{\Omega}\left|u_{\ell}(x)\right|^{2} d x=0,
\end{gathered}
$$

and $\lambda_{1}<\lambda_{2} \leq \cdots \lambda_{\ell} \leq \cdots$ is the sequence of all eigenvalues. That means that a concentration on a subset of $\Omega$ can occur only for an eigenvalue subsequence of density 0. A finer version can be stated in the language of semi-classical defect measures reviewed in the proof of Theorem 8 for a subsequence of density one the only limiting measure is the Liouville measure on the cosphere bundle.

Theorem 3 states that there cannot exists any sequence of eigenfunctions which concentrates away from a region geometrically controlling $\Omega \backslash R$, in particular, away from any neighbourhood of the vertical boundaries of the rectangular part. This is in agreement with the numerical (see for instance 11 and references given there) and experimental (for instance [11 and Figure 21) results indicating that there exist bouncing ball modes which on a sequence of density zero concentrate inside of the rectangle, stretching all the way to the boundary of the rectangle. Other applications, for instance to the case of the Sinai billiard, are presented in 10]. The fact that the semi-classical measure of any sequence of eigenfunctions (with increasing eigenvalues) is invariant by the action of the Hamiltonian flow is a consequence of the propagation results (Lemma 4.1). Apart from the beautiful, more precise but particular results on arithmetic surfaces [34], 31], this result seems to be the first one showing that there exist some invariant measures which are not the semi-classical measures of any sequence of eigenfunctions. 
We are going to deduce Theorem 3 from the following result [4] which is related to some earlier control results of Haraux [22] and Jaffard [27].

Proposition 6.1. Let $\Delta$ be the Dirichlet Laplace operator on the rectangle $R=$ $[0,1]_{x} \times[0, a]_{y}$. Then for any open nonempty $\omega \subset R$ of the form $\omega=\omega_{x} \times[0, a]_{y}$, there exists $C$ such that for any solutions of

$$
(\Delta-z) u=f \text { on } R,\left.u\right|_{\partial R}=0, z \in \mathbb{R}
$$

we have

$$
\|u\|_{L^{2}(R)}^{2} \leq C\left(\|f\|_{H^{-1}\left([0,1]_{x}, L^{2}\left([0, a]_{y}\right)\right)}^{2}+\left\|u \uparrow_{\omega}\right\|_{L^{2}(\omega)}^{2}\right) .
$$

Proof. We decompose $u, f$ in terms of the basis of $L^{2}([0, a])$ formed by the Dirichlet eigenfunctions $e_{k}(y)=\sqrt{2 / a} \sin (2 k \pi y / a)$,

$$
u(x, y)=\sum_{k} e_{k}(y) u_{k}(x), \quad f(x, y)=\sum_{k} e_{k}(y) f_{k}(x),
$$

and we get for $u_{k}, f_{k}$ the equation

$$
\left(\Delta_{x}-\left(z+(2 k \pi / a)^{2}\right)\right) u_{k}=f_{k}, \quad u_{k}(0)=u_{k}(1)=0 .
$$

Since $\omega_{x}$ controls geometrically $[0,1]$, a slight variant of [6.8) (or, in this simple case, a direct calculation) gives

$$
\left\|u_{k}\right\|_{L^{2}\left([0,1]_{x}\right)}^{2} \leq C\left(\left\|f_{k}\right\|_{H^{-1}\left([0,1]_{x}\right)}^{2}+\left\|u_{k} \uparrow_{\omega_{x}}\right\|_{L^{2}(\omega)}^{2}\right),
$$

and summing the squares on $k$, we get (6.17) 3

Proof of Theorem $3{ }^{\prime}$. Let us take $x, y$ as the coordinates on the stadium, so that $x$ is the longitudinal direction, $y$ the transversal direction, and the internal rectangle is $[0,1]_{x} \times[0, a]_{y}$. Let us then consider $u, f$ satisfying $(\Delta-z) u=f, u=0$ on the boundary of the stadium, and $\chi(x) \in C_{0}^{\infty}(0,1)$ equal to 1 on $[\varepsilon, 1-\varepsilon]$. Then $\chi(x) u(x, y)$ is a solution of

$$
(\Delta-z) \chi u=\chi f+[\Delta, \chi] u \quad \text { in } R
$$

with Dirichlet boundary conditions on $\partial R$. Applying Proposition 6.1, we get

$$
\|\chi u\|_{L^{2}(R)} \leq C\left(\|\chi f\|_{H_{x}^{-1}, L_{y}^{2}}+\left\|u \uparrow_{\omega_{\varepsilon}}\right\|_{L^{2}\left(\omega_{\varepsilon}\right)}\right)
$$

where $\omega_{\varepsilon}$ is a neighbourhood of the support of $\nabla \chi$. Consequently we get for $V$ a neighbourhood of $\Omega \backslash R$,

$$
\|u\|_{L^{2}(R)} \leq C\left(\|f\|_{L^{2}(R)}+\left\|u \uparrow_{V}\right\|_{L^{2}(V)}\right) .
$$

Finally, by standard propagation of semi-classical singularities as in subsection 6.1 we can replace $V$ by $\omega$ in (6.23).

\footnotetext{
${ }^{3}$ We remark that as noted in [4] the proof applies to any product manifold $M=M_{x} \times M_{y}$, and consequently Theorem $3^{\prime}$ holds also for that geometry as a black box.
} 
6.4. Semi-classical control with a prescribed loss. For completeness we propose a natural class of examples in which we expect $G(h)$ in Theorems 4 and 5 to be essentially any power of $h$ :

$$
G(h)=h^{-\alpha} \log (1 / h), \quad \alpha=\frac{m-1}{m+1}, \quad m=1,2, \cdots .
$$

For that, consider the following set of Schrödinger operators on $\mathbb{R}^{2}$ :

$$
P_{m}(h)=-h^{2} \Delta+x_{1}^{2}-x_{2}^{2 m}, \quad m \in \mathbb{N} .
$$

The Helffer-Sjöstrand theory of resonances [23] applies to this case (see also [35] Section 1] where a discussion of a general polynomial is given). In particular, for the meromorphically continued resolvent, $R_{m}(z, h)=\left(P_{m}(h)-z\right)^{-1}$, we have the following bound for the cut-off resolvent:

$$
\left\|\chi R_{m}(z, h) \chi\right\| \leq C h^{-\frac{2 m}{m+1}} \log (1 / h) .
$$

A separation of variables argument and the rescaling $x=h^{\frac{1}{m+1}} y$ suggest that the resonances are at the distance $h^{\frac{2 m}{m+1}}$ from the real axis. The same method shows that the resolvent is polynomially bounded in $h^{-1}$ and hence the interpolation argument we used before (see Lemma A.2 below) gives (6.24).

From $P_{m}(h)$ we can construct a "black box" for an operator $P(h)$ to which Theorems 4 and 5 will be applicable with $G(h)=h^{-\frac{m-1}{m+1}} \log (1 / h)$.

6.5. Closed hyperbolic orbits on manifolds. We will now discuss in more detail the case occurring when the black box contains a hyperbolic orbit, leading to the proof of Theorem 2 .

Thus suppose that the hypotheses of that theorem are satisfied. It is well known that we can find a coordinate system in a neighbourhood of $\gamma, U \simeq \mathbb{S}^{1} \times V, V$ a neighbourhood of 0 in $\mathbb{R}^{n-1}$, in which $\gamma$ is identified with $\mathbb{S}^{1}$ and the metric is given by

$$
g=d \theta^{2}+\sum_{1 \leq i, j \leq n-1} h_{i j}(x, \theta) d x_{i} d x_{j}, \quad \theta \in \mathbb{S}^{1}, x \in V .
$$

Since $\gamma$ is hyperbolic, we can assume that $\mathbb{S}^{1}$ is the only closed geodesic in $U$.

From this local construction we now build a global scattering problem by extending $g$ to a metric, $g_{\mathrm{bb}}$, defined on $\mathbb{S}^{1} \times \mathbb{R}^{n-1} \simeq \mathbb{S}_{\theta}^{1} \times \mathbb{S}_{\omega}^{n-1} \times[0, \infty)$. We choose $g$ to be asymptotically Euclidean:

$$
g_{\mathrm{bb}} \sim d r^{2}+r^{2} d \theta^{2}+r^{2} g_{\mathbb{S}^{n-1}}(d \omega), \quad r \rightarrow \infty,
$$

and so that $\gamma$ is the only closed geodesic of $g_{\mathrm{bb}}$.

Because of the work of Ikawa [26], Gérard [17], and of Gérard-Sjöstrand [18], it is expected that the resolvent of the Laplacian of $g_{\mathrm{bb}}$ can be controlled using (6.13), as in subsection 6.2 Since the two metrics agree in a neighbourhood of the closed geodecics, we can use the scattering problem as our "black box" and apply Theorem 5 with $A=(1-\chi)$. That would give Theorem 2 with $(\log \lambda)^{2}$ in place of $\log \lambda$. To get the improved (and, thanks to an example in [13, optimal) statement, we need an improved estimate for the resolvent so that Theorem 5 can be applied:

$$
\left\|\chi R_{\mathrm{bb}}(k) \phi\right\|_{L^{2} \rightarrow L^{2}} \leq C_{1} \frac{\sqrt{\log \langle k\rangle}}{\langle k\rangle}, \quad \phi \in \mathcal{C}_{\mathrm{c}}^{\infty}, \operatorname{supp} \phi \cap \gamma=\emptyset .
$$


Since the needed results from scattering theory, although expected, are not yet available 4 , we take a simplified route and use a complex absorbing potential to construct a black box operator $Q$ in Theorem $5^{5}$. That is done in the Appendix with Theorem A furnishing us with the needed estimates. Since the subprincipal symbol of the Laplace operator $-h^{2} \Delta$ is equal to 0 , Theorem 2 follows from its more general fully semi-classical variant:

Theorem 2. Suppose that $X$ is a compact $n$-manifold or $\mathbb{R}^{n}$ and that $P(h) \in$ $\Psi_{h}^{m, 0}\left(X, \Omega_{X}^{\frac{1}{2}}\right)$ has the real principal symbol, $p$, satisfying

$$
\begin{gathered}
p^{-1}([-\epsilon, \epsilon]) \Subset T^{*} X, \quad \text { for some } \epsilon>0, \\
p(\rho)=0 \Longrightarrow d p(\rho) \neq 0, \\
\exists C>0 \quad\langle\xi\rangle \geq C \Longrightarrow p \geq\langle\xi\rangle^{m} / C .
\end{gathered}
$$

Assume also that the subprincipal symbol of $p,(2.2)$, is equal to zero. Let $\gamma \subset$ $p^{-1}(0)$ be a closed hyperbolic orbit of the Hamilton flow of $p$, in the sense that all eigenvalues of the linearized Poincaré map are real and different from one.

There exist constants $C_{0}$ and $h_{0}$, such that if $u(h) \in L^{2}\left(X, \Omega_{X}^{\frac{1}{2}}\right)$ satisfies

$$
P(h) u=f,
$$

then for any $A(h) \in \Psi_{h}^{0,0}\left(X, \Omega_{X}^{\frac{1}{2}}\right)$, with its essential support, $W F(A)$, contained in a small neighbourhood of $\gamma$, we have

$$
C_{0}\left(h^{-2}(\log (1 / h))^{2} \int_{X}|f|^{2}+\log (1 / h) \int_{X}|(I-A(h)) u|^{2}\right) \geq \int_{X}|u|^{2}, \quad h<h_{0} .
$$

\section{APPENDIX}

In this appendix we will construct an operator $Q$ appearing in Theorem 5 for a black box containing a hyperbolic orbit on a Riemannian manifold, or more generally for the operator appearing in Theorem $2^{\prime}$. Ideally, we would like $Q$ to be the complex scaled Laplacian, $-h^{2} \Delta_{\theta}-z$ on an asymptotically Euclidean manifold having one closed hyperbolic geodesic as its trapped set. The results of [17, [18] indicate that precise estimates of the type needed, and in fact, the full understanding of resonances in logarithmic neighbourhoods of the real axis, should be possible.

In the case of the Laplacian on a compact Riemannian manifold we construct the reference operator as follows. Let $a \in \mathcal{C}^{\infty}(X,[0,1])$ be equal to 0 in a neighbourhood of $\gamma$ and to 1 in a neighbourhood of infinity. We then put

$$
Q=Q(z) \stackrel{\text { def }}{=}-h^{2} \Delta_{g}-z-i h a, \quad z \in[1,2]+i[-\epsilon, \epsilon] .
$$

When considering a more general operator $P(h)$ given in Theorem $2^{\prime}$, we put

$$
Q=Q(z) \stackrel{\text { def }}{=} P(h)-z-i h a, \quad z \in[1,2]+i[-\epsilon, \epsilon],
$$

where $a$ is a pseudodifferential operator with $W F_{h}(a) \cap \gamma=\emptyset$ and $a$ is elliptic, positive away from $\gamma$ on $T^{*} X$. We recall that $P(h)$ is assumed to be of real principal type and to have a vanishing subprincipal symbol. That means that the Weyl

\footnotetext{
${ }^{4}$ In [26] only convex obstacles in the Euclidean case are studied, while in [18] an analyticity assumption is made.

${ }^{5}$ We remark however that the results of [20] and [12] would have been sufficient for the case of hyperbolic geodesics on constant negative curvature segments, if one takes the black box approach.
} 
principal symbol of $P(h)$ is independent of $h$ and in any local coordinates $P(h)$ differs from its Weyl quantization by $\mathcal{O}\left(h^{2}\right)$.

The following result will allow applications of Theorem 5 :

Theorem A. If $Q(z)$ is given by A.1 and $z \in I \Subset(0, \infty)$, then for $h<h_{0}$, we have

$$
\left\|Q(z)^{-1}\right\|_{L^{2}(X) \rightarrow L^{2}(X)} \leq C \frac{\log (1 / h)}{h} .
$$

If $\phi \in \mathcal{C}_{\mathrm{b}}^{\infty}(X)$ is supported away from $\gamma$, then we also have

$$
\left\|Q(z)^{-1} \phi\right\|_{L^{2}(X) \rightarrow L^{2}(X)} \leq C \frac{\sqrt{\log (1 / h)}}{h} .
$$

To prove this theorem, we will use the strategy of the proof of Theorem 5 which means that it will be reduced to a local estimate near $\gamma$.

We first show that we have control away from a small neighbourhood of $\gamma$. See Figure 6 for an illustration of the hypotheses of the following

Proposition A.1. Suppose that $\psi_{0} \in S^{0}\left(T^{*} X\right) \cap \mathcal{C}_{\mathrm{c}}^{\infty}\left(T^{*} X\right)$ is a microlocal cutoff to a small neighbourhood of $\gamma \subset p^{-1}(0)$. Then, with $Q(z)$ as in (A.1) and $z \in[1,2]+i\left(-c_{0} h,+\infty\right), c_{0}>0$, we have

$$
Q(z) u=f \Longrightarrow\left\|\left(1-\psi_{0}\right) u\right\| \leq C \frac{1}{h}\|f\|+\mathcal{O}\left(h^{\infty}\right)\|u\| .
$$

Proof. We will prove A.2 using Lemma 4.1. For that we choose $c_{0}>0$ and $\psi_{1}, \psi_{2} \in \mathcal{C}_{\mathrm{c}}^{\infty}\left(T^{*} X\right)$ with $W F_{h}\left(1-\psi_{j}\right) \cap \gamma=\emptyset$, so that, as operators,

$$
\left(a-c_{0}\right)\left(1-\psi_{1}\right) \geq\left\{\begin{array}{l}
c_{0} / 2\left(1-\psi_{1}\right), \\
c_{0}\left(1-\psi_{2}\right)^{*}\left(1-\psi_{2}\right) / 2,
\end{array}\right.
$$

and $\psi_{1} \equiv 0$ on $\operatorname{supp}\left(1-\psi_{2}\right)$. We then have

$$
\begin{aligned}
\frac{1}{2} c_{0} h \int_{X}\left|\left(1-\psi_{2}\right) u\right|^{2} & \leq h \int_{X}(a+(\operatorname{Im} z / h)) \overline{u\left(1-\psi_{1}\right) u} \\
& =-\operatorname{Im} \int_{X} Q(z) u \overline{\left(1-\psi_{1}\right) u} \\
& =-\operatorname{Im} \int_{X} f \overline{\left(1-\psi_{1}\right) u} \\
& \leq\|f\|\left(\left\|\left(1-\psi_{1}\right) u\right\|+\mathcal{O}\left(h^{\infty}\right)\|u\|^{2}\right) \\
& \leq 2 \varepsilon h^{-1}\|f\|^{2}+\varepsilon h\left\|\left(1-\psi_{1}\right) u\right\|^{2}+\mathcal{O}\left(h^{\infty}\right)\|u\|^{2},
\end{aligned}
$$

where we use the same symbols to denote the operator Weyl quantizing the corresponding functions. Lemma 4.1 can be applied to $Q(z)$ since both the imaginary term $i a(x) h$ and $z$ are lower order terms, and we can choose $A u \stackrel{\text { def }}{=}\left(1-\psi_{2}\right) u$. Hence

$$
\begin{aligned}
\left\|\left(1-\psi_{1}\right) u\right\|^{2} & \leq C\|f\|\left(\frac{1}{h}\|f\|+\left\|\left(1-\psi_{2}\right) u\right\|+\mathcal{O}\left(h^{\infty}\right)\|u\|\right) \\
& \leq 2 \frac{C}{\varepsilon} h^{-1}\|f\|^{2}+C \varepsilon h\left\|\left(1-\psi_{2}\right) u\right\|^{2}+\mathcal{O}\left(h^{\infty}\right)\|u\|^{2},
\end{aligned}
$$

which proves (A.2) with $\psi_{0}$ replaced by $\psi_{1}$. We can estimate $\left\|\left(\psi_{0}-\psi_{1}\right) u\right\|$ using Lemma 4.1 which gives (A.2). 


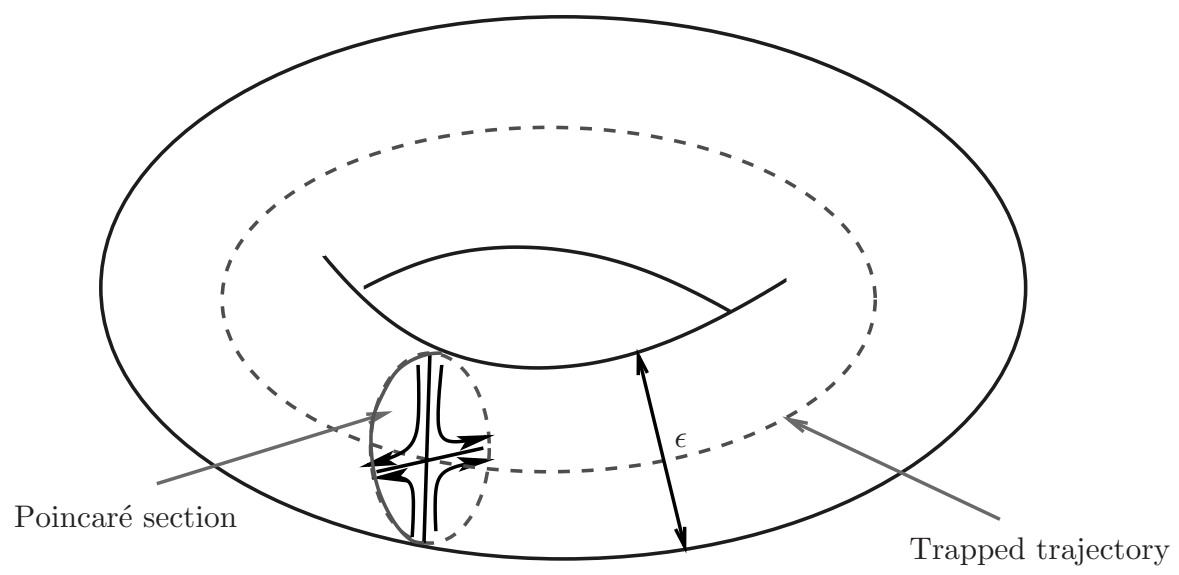

Figure 6. A hyperbolic trapped trajectory.

With the help of the above result we have essentially reduced the proof of Theorem A to the proof of the following

Proposition A.2. With the notation of Proposition A.1 there exist $c_{0}, h_{0}$, and $N_{0}$ such that for $u$ with $W F_{h}(u)$ in a small neighbourhood of $\gamma, z \in[1,2]+i\left(-c_{0} h,+\infty\right)$, and $h<h_{0}$, we have

$$
\|u\| \leq C h^{-N_{0}}\|Q(z) u\| .
$$

To prove this, we start with the following essentially well-know geometric lemma (see for instance [21]):

Lemma A.1. Suppose that $p \in \mathcal{C}^{\infty}\left(T^{*} X\right)$ satisfies $p=0 \Rightarrow d p \neq 0$ and that $\gamma \subset p^{-1}(0)$ is a closed real hyperbolic orbit of the Hamilton flow of $p$. There exists a neighbourhood $U$ of $\gamma$ in $T * X$ and a symplectomorphism $\kappa: U \rightarrow \kappa(U) \subset$ $T^{*} \mathbb{S}_{(t, \tau)}^{1} \times T^{*} \mathbb{R}_{(x, \xi)}^{n-1}$ such that

$$
\begin{aligned}
\kappa(\gamma) & =\left\{(t, 0 ; 0,0): t \in \mathbb{S}^{1}\right\}, \quad p=\kappa^{*}\left(h+h_{3}\right), \\
h(t, \tau ; x, \xi) & =\tau+\sum_{j=1}^{n-1} \lambda_{j} x_{j} \xi_{j}, \quad h_{3}(t, \tau, x, \xi)=\mathcal{O}\left(|x|^{3}+|\xi|^{3}\right) .
\end{aligned}
$$

The lemma is schematically illustrated in Figure 6 where the axes in the Poincaré section correspond to $x$ and $\xi$.

Proof of Proposition A.2 In the notation of Lemma A.1 we can assume that ess-supp $\psi_{0} \subset U$ and hence we will work microlocally in that neighbourhood which we identify with the neighbourhood of $\mathbb{S}^{1} \times\{(0 ; 0,0)\}$ in $T^{*} \mathbb{S}^{1} \times T^{*} \mathbb{R}^{n-1}$. The principal part of $P(h)$ is then of the form given in (A.3). We will use the same notation for the full symbol of $P(h)$ defined in these coordinates: $P(h)=\mathrm{Op}_{h}^{w}(p(\bullet, h))$.

We now follow a method inspired by many previous works on resonances generated by a hyperbolic trajectory; see [3, Section 6.3] for a recent presentation and references (among them [17] and [18], already mentioned above). The aim there was to prove the closely related $\mathcal{O}\left(h^{-C}\right)$ bounds on resonance projectors. Instead 
of using the FBI transform, we follow [14, Section 4.2] and make the presentation essentially self-contained.

We start with a rescaling in $(x, \xi)$ coordinates:

$$
X=(h M)^{-\frac{1}{2}} x, \quad \Xi=(h M)^{-\frac{1}{2}} \xi, \quad|(X, \Xi)| \leq(h M)^{-\frac{1}{2}} \epsilon,
$$

where $M$ is a large constant. Unless specifically stated, all estimates below are uniform in $M$ for $0<h<h_{0}(M)$. We note that the unitary transformation

$$
U_{h, M} u(X)=(h M)^{\frac{n-1}{2}} u\left((h M)^{\frac{1}{2}} X\right)
$$

conjugates operators $a^{w}\left(x, h D_{x}\right)$ and $a_{h, M}^{w}\left(X, M^{-1} D_{X}\right)$,

$$
a_{h, M}(X, \Xi)=a\left((h M)^{\frac{1}{2}} X,(h M)^{\frac{1}{2}} \Xi\right) .
$$

Hence,

$$
\begin{aligned}
\widetilde{P}(h) & =U_{h, M}^{-1} P(h) U_{h, M}=U_{h, M}^{-1} \mathrm{Op}_{h}^{w}\left(p^{w}\left(t, h D_{t}, \bullet, h\right)\right) U_{h, M} \\
& =\mathrm{Op}_{M^{-1}}^{w}\left(\tilde{p}^{w}\left(t, h D_{t}, \bullet, h\right)\right),
\end{aligned}
$$

where $\mathrm{Op}_{M^{-1}}^{w}$ denotes the Weyl quantization in $(X, \Xi)$ variables with $h$ replaced by $M^{-1}$, and, since the subprincipal symbol of $P(h)$ vanishes,

$$
\begin{gathered}
\tilde{p}(t, \tau ; X, \Xi, h)=\tau+\sum_{j=1}^{n-1} M h \lambda_{j} X_{j} \Xi_{j}+(h M)^{\frac{3}{2}} \mathcal{O}\left(|X|^{3}+|\Xi|^{3}\right)+\mathcal{O}\left(h^{2}\right), \\
\left|\partial_{X, \Xi}^{\alpha} \tilde{p}\right| \leq C_{\alpha}\left((h M)^{\frac{1}{2}}+\epsilon /\langle(X, \Xi)\rangle\right)^{|\alpha|} .
\end{gathered}
$$

In particular, the remainder, $\mathcal{O}\left(h^{2}\right)$, satisfies these estimates multiplied by $h^{2}$.

Let $\psi_{0} \in \mathcal{C}_{\mathrm{c}}^{\infty}\left(T^{*} \mathbb{R}^{n-1}\right)$ be supported near $(0,0)$ and equal to 1 in a smaller neighbourhood of that point. In variables $X$ and $\Xi$ we define the escape function

$$
\begin{gathered}
G(X, \Xi) \stackrel{\text { def }}{=} \frac{1}{2} \sum_{j=1}^{n-1}\left(\log \left(1+X_{j}^{2}\right)-\log \left(1+\Xi_{j}^{2}\right)\right) \psi_{0}\left(h^{\frac{1}{2}}(X, \Xi)\right), \\
\left|\partial_{X}^{\alpha} \partial_{\Xi}^{\beta} G(X, \Xi)\right| \leq C_{\alpha \beta}\langle X\rangle^{-|\alpha|}\langle\Xi\rangle^{-|\beta|}, \quad(0,0) \neq(\alpha, \beta) \in \mathbb{N}^{2(n-1)},
\end{gathered}
$$

and also

$$
G=\mathcal{O}(\log (1 / h)) .
$$

For $|(X, \Xi)| \leq h^{-\frac{1}{2}} \epsilon$ we also have, using (A.6),

$$
\begin{aligned}
\frac{1}{h M} H_{\tilde{p}} G= & \frac{1}{h M} \sum_{j=1}^{n-1}\left(\frac{X_{j} \partial_{\Xi_{j}}}{1+X_{j}^{2}}+\frac{\Xi \partial_{X_{j}}}{1+\Xi_{j}^{2}}\right) \\
& \times\left(\sum_{j=1}^{n-1} M h \lambda_{j} X_{j} \Xi_{j}+(h M)^{\frac{3}{2}} \mathcal{O}\left(|X|^{3}+|\Xi|^{3}\right)+\mathcal{O}\left(h^{2}\right)\right) \\
= & \sum_{j=1}^{n-1}\left(\frac{\lambda_{j} X_{j}^{2}}{1+X_{j}^{2}}+\frac{\lambda_{j} \Xi_{j}^{2}}{1+\Xi_{j}^{2}}\right)(1+\mathcal{O}(\epsilon))+\mathcal{O}\left(h^{\frac{3}{2}} M^{-\frac{1}{2}}\right) .
\end{aligned}
$$

We now consider $G^{w}=\mathrm{Op}_{M^{-1}}^{w}(G)$ and define

$$
\widetilde{P}_{t}(h)=e^{t G^{w}} \widetilde{P}(h) \operatorname{Op}_{M^{-1}}^{w}\left(\psi_{0}\left(h^{\frac{1}{2}} \bullet\right)\right) e^{-t G^{w}}=\exp \left(\operatorname{tad}_{G^{w}}\right) \widetilde{P}(h) \operatorname{Op}_{M^{-1}}^{w}\left(\psi_{0}\left(h^{\frac{1}{2}} \bullet\right)\right),
$$

with the exponential defined in the sense of bounded self-adjoint operators. 
The Weyl calculus, with the effective Planck constant given by $M^{-1}$, and using the estimates in (A.6) and (A.7), gives

$$
\left[\widetilde{P}(h), G^{w}\right]=\frac{1}{i M} \mathrm{Op}_{M^{-1}}^{w}\left(H_{\tilde{p}} G\right)+\mathcal{O}\left(M^{-2} h\right) .
$$

In fact, the composition formula holds:

$$
\begin{gathered}
\mathrm{Op}_{M^{-1}}^{w}(a) \circ \mathrm{Op}_{M^{-1}}^{w}(b)=\mathrm{Op}_{M^{-1}}^{w}(a \sharp b), \\
a \sharp b(x, \xi) \sim \sum_{k=0}^{\infty} \frac{1}{k ! M^{k}}\left(\sigma\left(D_{x}, D_{\xi} ; D_{y}, D_{\eta}\right)\right)^{k} a(x, \xi) b(y, \eta) \Gamma_{(x, \xi)=(y, \eta)},
\end{gathered}
$$

and the estimates of the remainders follow from the estimates on the individual terms; see [15, Chapter 7]. Similarly, for $h$ small enough and $M$ large and fixed,

$$
\begin{gathered}
\widetilde{P}_{t}(h)=\exp \left(\operatorname{tad}_{G^{w}}\right) \widetilde{P}(h) \operatorname{Op}_{M^{-1}}^{w}\left(\psi_{0}\left(h^{\frac{1}{2}} \bullet\right)\right) \sim \sum_{k=0}^{\infty} \frac{t^{k}}{k !} \operatorname{ad}_{G^{w}}^{k}\left(\widetilde{P}(h) \operatorname{Op}_{M^{-1}}^{w}\left(\psi_{0}\left(h^{\frac{1}{2}} \bullet\right)\right)\right), \\
\operatorname{ad}_{G^{w}}^{k}\left(\widetilde{P}(h) \operatorname{Op}_{M^{-1}}^{w}\left(\psi_{0}\left(h^{\frac{1}{2}} \bullet\right)\right)\right)=\mathcal{O}_{L^{2} \rightarrow L^{2}}\left(h M^{1-k}\right) .
\end{gathered}
$$

Hence, using (A.8),

$$
\widetilde{P}_{t}(h)=\widetilde{P}(h)-i t h A^{w}+t h \mathcal{O}\left(h+M^{-2}\right)+\mathcal{O}\left(t^{2} h M^{-1}\right), \quad A^{w}=\mathrm{Op}_{M^{-1}}^{w}(A),
$$

where $\mathcal{O}$ is meant in the sense of operators on $L^{2}$ and

$$
A(X, \Xi)=\sum_{j=1}^{n-1}\left(\frac{\lambda_{j} X_{j}^{2}}{1+X_{j}^{2}}+\frac{\lambda_{j} \Xi_{j}^{2}}{1+\Xi_{j}^{2}}\right)(1+\mathcal{O}(\epsilon))
$$

We will now show that for $M$ sufficiently large,

$$
\left\langle A^{w} U, U\right\rangle \geq \frac{c_{0}}{M}\|U\|^{2}
$$

which essentially is the lower bound for the harmonic oscillator $\left(D_{X} / M\right)^{2}+X^{2}$. In fact, assuming for simplicity $\lambda_{j}=1$ and using the $M^{-1}$-Weyl calculus, we write

$$
\begin{gathered}
A_{0}(X, \Xi)=\frac{X^{2}}{\langle X\rangle^{2}}+\frac{\Xi^{2}}{\langle\Xi\rangle^{2}}=|B(X, \Xi)|^{2}, \quad B=\frac{X}{\langle X\rangle}-i \frac{\Xi}{\langle\Xi\rangle}, \\
A_{0}^{w}\left(X, M^{-1} D_{X}\right)=B^{w}\left(X, M^{-1} D_{X}\right)^{*} B^{w}\left(X, M^{-1} D_{X}\right)+C^{w}\left(X, M^{-1} D_{X}, M^{-1}\right), \\
C\left(X, \Xi, M^{-1}\right)=M^{-1}\langle X\rangle^{-3}\langle\Xi\rangle^{-3}+\mathcal{O}\left(M^{-2}\right) .
\end{gathered}
$$

The $C^{w}$ term provides control for $(X, \Xi)$ small and the $\left(B^{w}\right)^{*} B^{w}$ term gives a lower bound (independent of $M$ ) in the rest of phase space, giving (A.10 with $A$ replaced by $A_{0}$. Since the operators differ by an elliptic factor, invertible for $M$ large, A.10) follows.

Hence for $U$ satisfying $\operatorname{Op}_{M^{-1}}^{w}\left(\psi_{0}\left(h^{\frac{1}{2}} \bullet\right)\right) U=U+\mathcal{O}\left(h^{\infty}\right)$ we see that

$$
-\operatorname{Im}\left\langle\widetilde{P}_{t}(h) U, U\right\rangle \geq \frac{h}{C_{1} M}\|U\|^{2} .
$$

Translating this to estimates for the original operator shows that for $u$ with $W F_{h}(u)$ in a small neighbourhood of $\gamma$, we have

$$
-\operatorname{Im}\left\langle e^{t B_{h}}(P(h)-z) e^{-t B_{h}} u, u\right\rangle \geq \frac{h}{C_{2}}\|u\|^{2}, \quad z \in[1,2]+i\left(-c_{0} h,+\infty\right),
$$


where $B_{h}=\mathcal{O}(\log (1 / h))$ comes from transplanting $G^{w}$ to the original coordinates by the unitary transformation described after (A.4). Since $\left\|\exp \left( \pm t B_{h}\right)\right\|=\mathcal{O}\left(h^{-N}\right)$ for some $N$, the proposition follows.

To prove Theorem A, we need the following lemma which, for possible future use, we state in a slightly excessive generality:

Lemma A.2. Suppose that $A$ and $B$ are bounded self-adjoint operators on a Hilbert space $\mathcal{H}$,

$$
A^{2}=A, \quad B A=A B=A,
$$

and $F(z)$ is a family of bounded operators satisfying

$$
\begin{gathered}
F(z)^{*}=F(\bar{z}), \quad \partial_{z} F \uparrow_{\mathbb{R}} \geq c I d, c>0, \\
B F(z)^{-1} B \text { is holomorphic in }[-\epsilon, \epsilon]+i[-\delta, \delta], \quad \frac{\delta}{\epsilon} \ll 1 / \log M, \\
\left\|B F(z)^{-1} B\right\| \leq M, \quad\left\|A F(z)^{-1} A\right\| \leq 1 .
\end{gathered}
$$

Then for $|z|<\epsilon / 2, \operatorname{Im} z=0$ we have

$$
\left\|B F(z)^{-1} B\right\| \leq C \frac{\log M}{\delta}, \quad\left\|B F(z)^{-1} A\right\| \leq C \sqrt{\frac{\log M}{\delta}} .
$$

Proof. The first part of (A.12) works exactly as in [40. Lemma 2] and [7, Lemma 4.2]. To see the improved version, we start by observing that the conditions on $F$ and $A$ imply that for $\operatorname{Im} z>0$, small,

$$
\operatorname{Im} z\|u\|^{2} \leq C \operatorname{Im}\langle F(z) u, u\rangle .
$$

If now $F(z) u=A f$, then by the assumptions on $F,\|A u\| \leq\|A f\|$, and consequently,

$$
\|B u\|^{2} \leq C\|u\|^{2} \leq \frac{1}{\operatorname{Im} z}\langle A f, A u\rangle \leq \frac{1}{\operatorname{Im} z}\|A f\|^{2} .
$$

Here we used the facts that $A^{2}=A=A^{*}$. Since $u=F(z)^{-1} A f$, this and the fact that $B A=A$ give

$$
\begin{aligned}
& \left\|B F(z)^{-1} A\right\| \leq \frac{C}{\sqrt{\operatorname{Im} z}}, \quad \operatorname{Im} z>0, \\
& \left\|B F(z)^{-1} A\right\| \leq C\left\|B F(z)^{-1} B\right\| \leq C M .
\end{aligned}
$$

Interpolating as before gives (A.12).

Proof of Theorem A. We first combine Propositions A.1 and A.2 to see that

$$
\begin{gathered}
\left\|\left(1-\psi_{0}\right) u\right\| \leq C h^{-1}\|Q u\|+\mathcal{O}\left(h^{\infty}\right)\|u\|, \\
\left\|\psi_{0} u\right\| \leq C h^{-N}\left\|\psi_{0} Q u\right\|+C h^{-N}\left\|\left[Q, \psi_{0}\right] u\right\| \leq C^{\prime} h^{-N}\|Q u\|+\mathcal{O}\left(h^{\infty}\right)\|u\|,
\end{gathered}
$$

where we used the fact that $\left\|\left[Q, \psi_{0}\right] u\right\|=\left\|\left[Q, \psi_{0}\right]\left(1-\widetilde{\psi}_{0}\right) u\right\|+\mathcal{O}\left(h^{\infty}\right)\|u\|$, for some $\tilde{\psi}_{0}$ satisfying the assumptions of Proposition A.1.

Finally we combine this latter estimate and (A.2) with Lemma A.2 applied to the family of operators $w \mapsto F(w)=(i / h) Q\left(z_{0}+h w\right)$ and $A=\mathbb{1}_{\text {supp } \phi}, B=1$. We can take $\delta$ independent of $h$ and $\epsilon=1 /(C h)$ so that the assumption $\delta / \epsilon \ll 1 / \log \left(1 / h^{N}\right)$ is satisfied. 


\section{ACKNOWLEDGMENTS}

We would like to thank the National Science Foundation for partial support under the grant DMS-0200732. We are also grateful to Steve Zelditch for informing us of [16] and [42] which expanded the breadth of this note, to Luc Miller for helpful comments on the first version of the paper, and to Victor Humphrey and Paul Chinnery for the permission to use their Figure 2 The first author thanks the Mathematical Science Research Institute for its hospitality during spring 2003.

Finally, we would like to express our thanks to four anonymous referees whose detailed comments lead to many improvements and corrections. We are particularly appreciative to the first referee's insistence that the result presented in the Appendix should have a direct and self-contained proof.

\section{REFERENCES}

[1] A. Bäcker, R. Schubert, and P. Stifter. On the number of bouncing ball modes in billiards. J. Phys. A: Math. Gen. 30:6783-6795, 1997.

[2] C. Bardos, G. Lebeau, and J. Rauch. Sharp sufficient conditions for the observation, control, and stabilization of waves from the boundary. SIAM J. Control Optim. 30:1024-1065, 1992. MR 94b:93067

[3] J.-F. Bony and L. Michel. Microlocalization of resonant states and estimates of the residue of scattering amplitude, Comm. Math. Phys., to appear.

[4] N. Burq. Control for Schrodinger equations on product manifolds. Unpublished, 1992.

[5] N. Burq. Contrôle de l'équation des plaques en présence d'obstacles strictement convexes. Mémoire de la S.M.F., 55, 1993. Supplément au Bulletin de la Société Mathématique de France. MR 95d:93007

[6] N. Burq. Semi-classical estimates for the resolvent in non trapping geometries. Int. Math. Res. Notices 5:221-241, 2002. MR 2002k:81069

[7] N. Burq. Smoothing effect for Schrödinger boundary value problems. Preprint, 2002.

[8] N. Burq and P. Gérard, Condition nécessaire et suffisante pour la contrôlabilité exacte des ondes, Comptes Rendus de L'Académie des Sciences, 749-752, t. 325, Série I, 1996. MR 98j:93052

[9] N. Burq and and G. Lebeau. Mesures de défaut de compacité, application au système de Lamé, Ann. Sci. École Norm. Sup. (4), No. 34, 817-870, 2001. MR 2003a:35186

[10] N. Burq and M. Zworski. Eigenfunctions for partially rectangular billiards. math. SP/0312098.

[11] P.A. Chinnery and V.F. Humphrey. Experimental visualization of acoustic resonances within a stadium-shaped cavity. Physical Review E, 53, 1996, 272-276.

[12] T. Christiansen and M. Zworski. Resonance wave expansions: two hyperbolic examples. Comm. Math. Phys. 212:323-336, 2000. MR 2001j:58050

[13] Y. Colin de Verdière and B. Parisse. Équilibre instable en régime semi-classique. I. Concentration microlocale. Comm. Partial Differential Equations, 9-10, 19, 1535-1563, 1994. MR 96b:58112

[14] N. Dencker, J. Sjöstrand, and M. Zworski. Pseudospectra of semiclassical (pseudo)differential operators. Comm. Pure Appl. Math. 57:384-415, 2004.

[15] M. Dimassi and J. Sjöstrand. Spectral asymptotics in the semiclassical limit. Cambridge Universtity Press, 1999. MR 2001b:35237

[16] H. Donnelly. Quantum unique ergodicity. Proc. Amer. Math. Soc. 131, 2945-2951, 2003.

[17] Ch. Gérard. Asymptotique des pôles de la matrice de scattering pour deux obstacles strictement convexes. Mém. Soc. Math. France (N.S.) 31 (1988), 146 pp. MR 91e:35168

[18] Ch. Gérard and J. Sjöstrand. Resonances en limite semiclassique et exposants de Lyapunov, Comm. Math. Phys. 116-2, 193-213, 1988. MR 89f:35057

[19] P. Gérard and E. Leichtnam, Ergodic Properties of Eigenfunctions for the Dirichlet Problem, Duke Math. Jour. 71, 559-607, 1993. MR 94i:35146

[20] L. Guillopé. Sur la distribution des longueurs des géodésiques fermées d'une surface compacte à bord totalement géodésique. Duke Math. J. 53:827-848, 1986. MR 88e:11042

[21] J.P. Françoise and V. Guillemin. On the period spectrum of a symplectic mapping, J. Funct. Anal. 100:317-358, 1993. MR 92j:58083 
[22] A. Haraux. Séries lacunaires et contrôle semi-interne des vibrations d'une plaque rectangulaire, J. Math. Pures Appl. 68-4:457-465, 1989. MR 91e:93039

[23] B. Helffer and J. Sjöstrand. Resonances en limite semi-classique, Mémoire de la S.M.F., 114, 1986. MR 88i:81025

[24] L. Hörmander. The Analysis of Linear Partial Differential Operators. Vol. III, IV. SpringerVerlag, Berlin, 1985. MR 87d:35002a MR 87d:35002b

[25] A. Iantchenko, J. Sjöstrand, and M. Zworski. Birkhoff normal forms in semi-classical inverse problems. Math. Res. Lett. 9:337-362, 2002. MR 2003f:35284

[26] M. Ikawa. Decay of solution of the wave equation in the exterior of several convex bodies. Annales de l'Institut Fourier 38(2):113-146, 1988. MR 90a:35028

[27] S. Jaffard Contrôle interne exact des vibrations d'une plaque rectangulaire. Portugal. Math. 47 (1990), no. 4, 423-429. MR 91j:93051

[28] J.P. Kahane. Pseudo-périodicité et séries de Fourier lacunaires. Annales Sc. de l'Ecole Normale Supérieure 79, 1962. MR 27:4019]

[29] G. Lebeau. Contrôle de l'équation de Schrödinger. Journal de Mathématiques Pures et Appl. 71:267-291, 1992. MR 93i:35018

[30] J.L. Lions. Contrôlabilité exacte, perturbation et stabilisation des systèmes distribués, R.M.A., Masson, 1988. MR 90a:49040

[31] E. Lindenstrauss. Invariant measures and arithmetic quantum unique ergodicity, preprint, 2003.

[32] R.B. Melrose and J. Sjöstrand. Singularities of Boundary Value Problems I and II, Comm. in Pure Appl. Math. 31 and 35, 593-617 and 129-168, 1978 and 1982. MR 58:11859. MR 83h:35120

[33] L. Miller. How violent are fast controls for Schrödinger equation? preprint, 2003.

[34] P. Sarnak. Arithmetic quantum chaos. In The Schur lectures (1992) (Tel Aviv), volume 8 of Israel Math. Conf. Proc., pages 183-236. Bar-Ilan Univ., Ramat Gan, 1995. MR 96d:11059

[35] J. Sjöstrand. Geometric bounds on the density of resonances for semiclassical problems, Duke Math. J. 60:1-57, 1990. MR 91e:35166

[36] J. Sjöstrand. A trace formula and review of some estimates for resonances. In Microlocal Analysis and Spectral Theory, volume 490 of NATO ASI series C, pages 377-437. Kluwer, 1997. MR 99e:47064

[37] J. Sjöstrand and M. Zworski. Complex scaling and the distribution of scattering poles. Journal of the A.M.S. 4(4):729-769, 1991. MR 92g:35166

[38] J. Sjöstrand and M. Zworski. Quantum monodromy and semiclassical trace formulæ. Journal d'Mathématiques Pures et Appl. 81:1-33, 2002.

[39] J.A.K. Suykens and J. Vandewalle (Eds.). Nonlinear Modeling: advanced black-box techniques, Kluwer Academic Publishers Boston, June 1998.

[40] S.H. Tang and M. Zworski. From quasimodes to resonances. Math. Res. Lett. 5:261-272, 1998. MR 99i: 47088

[41] J. Wunsch and M. Zworski. Distribution of resonances for asymptotically Euclidean manifolds. J. Diff. Geom. 55:43-82, 2000. MR 2002e:58062

[42] S. Zelditch. Quantum unique ergodicity. math-ph/0301035.

[43] S. Zelditch and M. Zworski, Ergodicity of eigenfunctions for ergodic billiards. Comm. Math. Phys. 175:673-682, 1996. MR 97a:58193

[44] E. Zuazua. Contrôlabilité exacte en temps arbitrairement petit de quelques modèles de plaques. Appendix A.1 to [30]. MR 90a:49040

Université Paris Sud, Mathématiques, Bât 425, 91405 Orsay Cedex, France

E-mail address: Nicolas.burq@math.u-psud.fr

Mathematics Department, University of California, Evans Hall, Berkeley, CaliforNIA 94720

E-mail address: zworski@math.berkeley.edu 